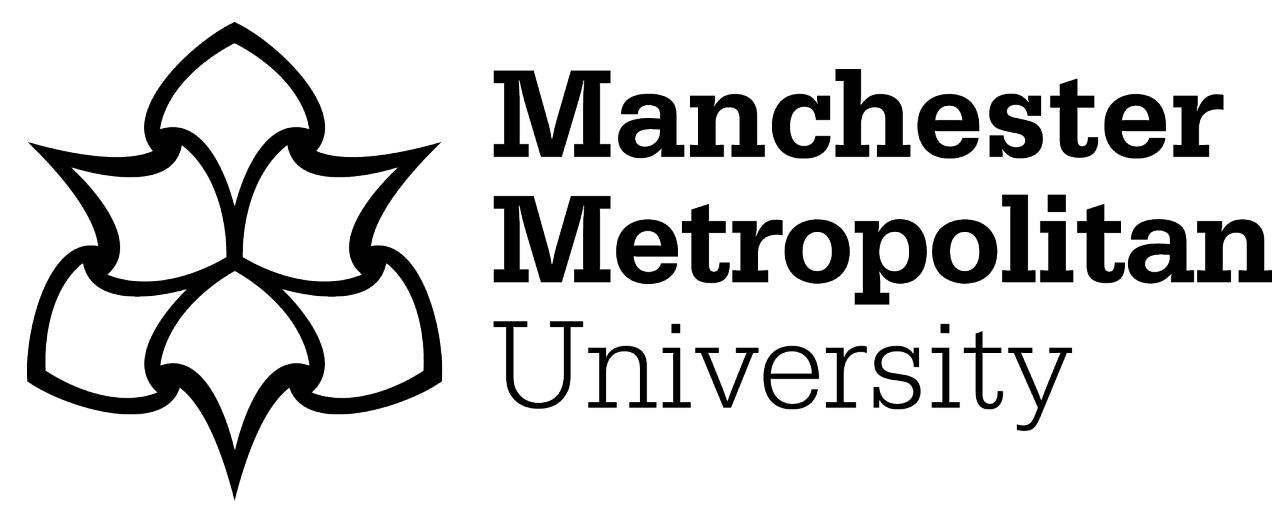

Khan, S, Kifayat, K, Kashif Bashir, A, Gurtov, A and Hassan, M (2021) Intelligent intrusion detection system in smart grid using computational intelligence and machine learning. Transactions on Emerging Telecommunications Technologies, 32 (6). e4062. ISSN 2161-3915

Downloaded from: https://e-space.mmu.ac.uk/627624/

Version: Accepted Version

Publisher: Wiley

DOI: https://doi.org/10.1002/ett.4062

Please cite the published version 


\title{
Intelligent Intrusion Detection System in Smart Grid Using Computational Intelligence and Machine Learning
}

\author{
Suleman Khan $^{1 *}$ | Kashif Kifayat ${ }^{1}$ | Ali Kashif Bashir, \\ SMIEEE $^{2}$ | Andrei Gurtov, SMIEEE ${ }^{3}$ | Mehdi Hassan ${ }^{1}$
}

${ }^{1}$ Air University, Islamabad, Pakistan

${ }^{2}$ School of Computing, Mathematics, and Digital Technology, Manchester Metropolitan University, United Kingdom

${ }^{3}$ Department of Computer and Information Science, Linköping University, Sweden

\section{Correspondence}

Suleman Khan, National Centre for Cyber Security (NCCS), Air University, Islamabad, Pakistan

Email: 171518@students.au.edu.pk

Funding information

Abbreviations: $A B C$, a black cat; $D E F$, doesn't ever fret; $G H I$, goes home immediately.
Smart grid systems enhanced the capability of traditional power networks while being vulnerable to different types of cyber-attacks. These vulnerabilities could cause attackers to crash into the network breaching the integrity and confidentiality of the smart grid systems. Therefore, an intrusion detection system (IDS) becomes an important way to provide a secure and reliable services in a smart grid environment. This paper proposes a feature-based IDS for smart grid systems. The proposed system performance is evaluated in terms of accuracy, intrusion detection rate and false alarm rate. The obtained results show that the Random Forest and Neural Network classifiers have outperformed other classifiers. We have achieved a $0.5 \%$ false alarm rate on KDD99 dataset and a $0.08 \%$ false alarm rate on the NSLKDD dataset. The detection rate and the testing accuracy on average are $99 \%$ for both datasets.

\section{KEYWORDS}

IoT,Smart Grids, Cyber Physical Systems, Cyber Security, Energy, Edge, Machine Learning, KDD99, NSLKDD 


\section{1 | INTRODUCTION}

Data-driven technologies is now applied to smart grid as a way of sustainable energy environment. This approach can be added to a cyber-physical system consisting of hardware, software and other physical gears. Smart grid supplies electricity on-demand to end-users from centralized stations and distribute to generating stations using information and communication technologies. Energy supplier companies supply electricity at low cost and also control the enduser demand for supply. In the smart grid system, one of the significant issues is security. Many vulnerabilities exist in cyber-physical systems and hackers take advantage of vulnerabilities to launch malicious attacks on power systems. Security problems usually include authentication, data protection, availability, confidentiality, honesty, energy efficiency, single-point failures to be tested, and more [1].The attackers destroy a whole range of cyberspace in modern electronic warfare. In our societies cybercrimes proliferated. Attacks, hacking, and malicious practices such as viruses, trojans, and spamming are common risks to individuals and nations. The digital networks of cellular telephony, wireless sensor networks, satellites, tactical military communications, Internet of Things, smart grids and Supervisory Control and Data Acquisition (SCADA) are everything vulnerable to that kind of electronic attack [2].A lot of work has been done on smart grid system implementation but the majority of work are not focusing on the security requirements for the smart grid systems [3, 4]. Intrusion detection system (IDS) plays an essential role in cyber-attacks on smart grid systems and secures them against attacks. The IDS are part of the network security domain and play a vital role in protecting and maintaining a secure network.IDS system is represented in figure 2.

A typical IDS system examines and analyzes network traffic to detect and analyze attacks, and also to prevent any security violations by generating alarms for network administrator. There are two major types of IDS: Host-based IDS and Network-based IDS. IDS can be further classified into Anomaly-based and Signature-based IDS systems [5, 6, 7]. Anomaly-based IDS detects attacks using previously recorded normal real-time traffic image and by comparing it with current traffic. Though, it is widely used in various IDS, it registers a large number of false-positive alarms [8,9]. The Signature-based IDS uses pattern matching with predefined signatures taken from the already detected malware's stored in a database. Thus, creating a low number of false positive alarms but at the same time, it lets new attacks to pass-through unnoticed $[10,11,12]$. Therefore, a system needs to be developed that can increase detection rate for new (a.k.a.zero-day malware's) attacks and reduce false alarms rate in previously defined signatures.

Figure 1 depicts the interaction between the power generation units, distribution centers and other different entities such as industries, smart buildings, households, etc. The smart grid plays a major role in efficiently dissipating the right amount of power to these various entities. The flexibility in the power distribution process is achieved by means of implementing various Al algorithms in the smart grid. The flexibility comes into picture due to the dynamic power requirement from various sectors.

This research uses optimized feature selection technique to detect and classify network intrusions using Signaturebased IDS while reducing false alarm rate.Typically, real-time traffic and patterns contain high dimensional space of features. Therefore, feature selection is commonly used to reduce the dimensionality in order to simplify a data set and identify relevant features without sacrificing predictive accuracy. An efficient feature selection can help in cleaning the real-time traffic from noise and irrelevant features $[13,14,15]$. Particle Swarm Optimization (PSO) is a commonly used technique for feature selection $[16,17,18]$. Easy to encode features, support for global searching, requirement of less computational power, fewer parameters and ease of use makes it a common choice of researchers [19, 20, 21]. Therefore, we have used PSO for feature selection in our experiments as well.

Machine-learning algorithms have been commonly used to detect and identify various types of attacks. In this paper, we have implemented several machine-learning algorithms to classify network packets into malicious or normal packets. The novel contribution of this research includes: Modification in the weights of particle swarm optimization 

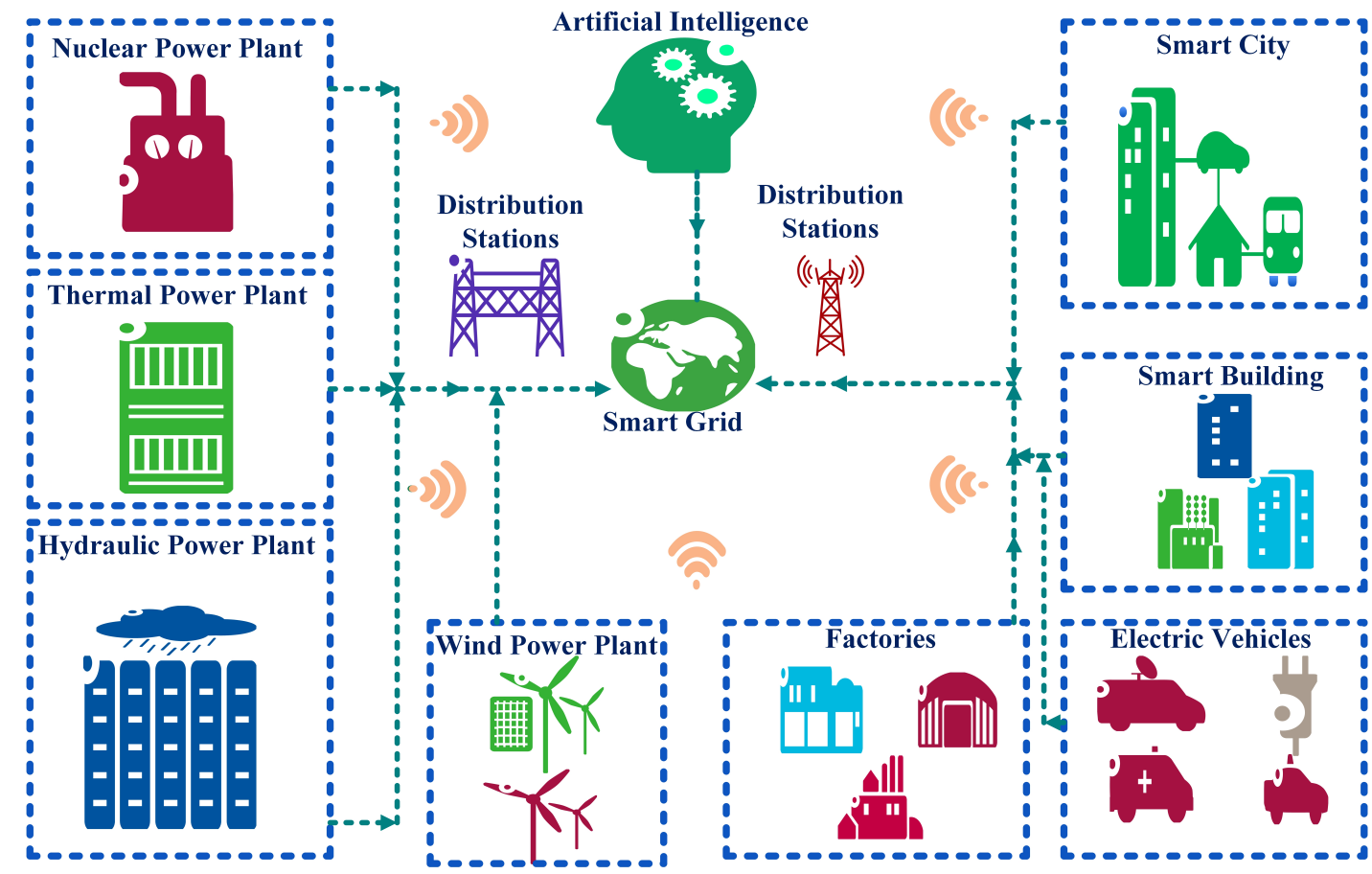

FIGURE 1 Smartgrid System Illustration

algorithms, allowing our proposed weighted particle swarm optimizer to select best features from data sets and those optimal features produces high detection rate, high accuracy and improved false alarm rate. In this research two data sets are used NSLKDD [22] and KDD99 [23]. After selection of data sets some prepossessing techniques are applied on both the data sets. Data sets are normalized using min-max normalization technique in order to scale the data. After data normalization data encoding is performed to convert nominal values to numeric values because machine learning works on numeric data. The proposed system performance is evaluated in terms of accuracy,intrusion detection rate and false alarm rate. The obtained results show that the Random Forest and a Neural Network classifiers have performed better. We have achieved a $0.5 \%$ false alarm rate on KDD99 and a $0.08 \%$ false alarm rate on the NSLKDD dataset. The detection rate and the testing accuracy on average are $99 \%$ for both datasets.

Paper Organization: Section II evaluates the existing studies and their possible limitations. Section III describes the proposed methodology and techniques adopted, followed by the experiments performed and results tabulated in Section IV. Section $V$ concludes the paper.

\section{2 | RELATED WORK}

The demand for electricity is rising day by day and it is estimated that electricity will increase by 30 to 40 percent over the next 20 years. Current power grids are very old; becoming more and more overloaded, unreliable and does not produce enough of electricity. A smart grid has an analytical and well-organized approach to the management of energy supply and usage. The smart grid tracks and regulates the flow of energy in two ways. The consumers 


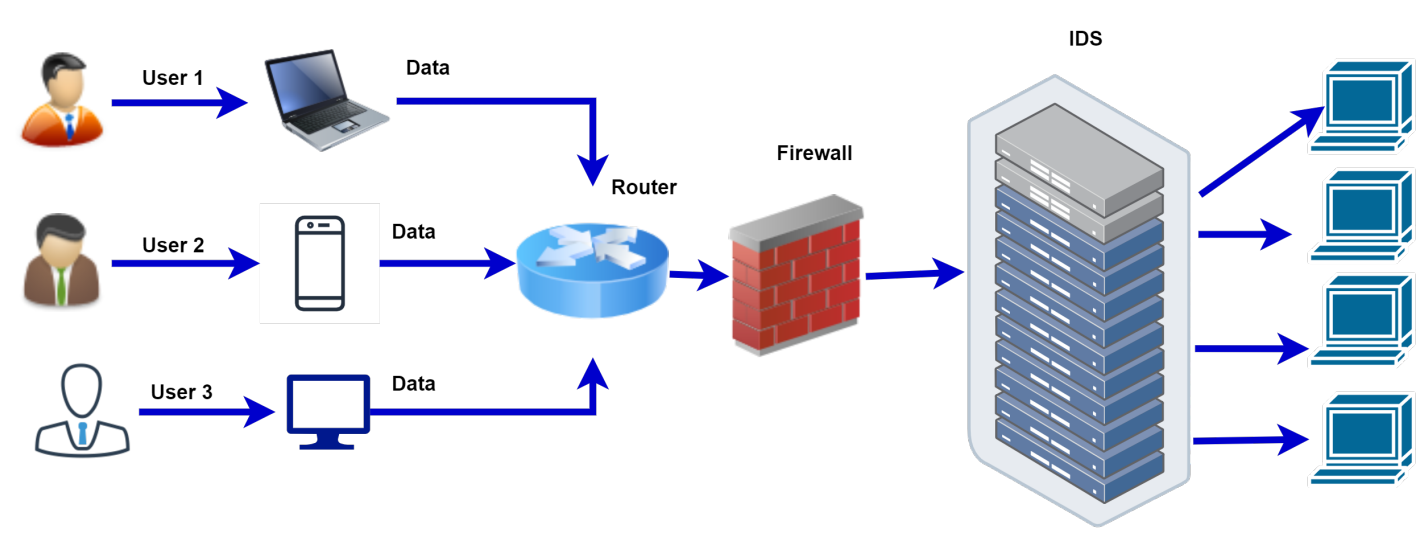

FIGURE 2 Intrusion detection system working

also had the option to use an optimized algorithm to buy the cheapest energy at a particular time, depending on the amount of power used.The smart grid facilitates bidirectional contact between energy suppliers and their clients. The transformation from the current power grid to the smart grid requires new funding, which guarantees the returned great value. The smart grid needs reliable, stable, cost-effective, efficient, environmentally sustainable and healthier facilities.

The smart grid has the below seven key features: allow active customer involvement; manage all production and storage options; create new products, utilities, and markets; offer the best digital economy with power reliability; use energy, optimization, and reliability; ability to self-heal and robust cyber and physical attack actions. The development of smart grids required the integration of diverse technologies and applications. The smart grid has four milestones: customer allowing, advanced delivery operations, advanced transmission operations, advanced asset management. By improving network-wide reliability and dynamic performance, the smart grid increases monitoring and control of the power system co-ordinates. Cyber protection is essential for automatic electric power system operation.

One of the first attempts to achieve a high detection rate and a reduced false alarm rate has been performed on the DARPA 1998 dataset [24].In this paper, authors have used Principal Component Analysis (PCA) to select features and neural networks for classification. Though PCA provides an optimal feature set, it compromises the training efficiency with correct results [25]. Another method for optimal feature selection has been used is Feature Vitality Based Reduction Method (FVBRM) algorithm [19]. The experiment has used 41 features on the NSLKDD dataset using the Naïve Bayes classifier. Some experiments have used multiple techniques for feature selection. Hee-Su et al. [26] have used four feature selection techniques. These techniques are Gain Ratio (GR), Correlation-based Feature Selection (CFS), Information Gain (IG) and Attribute Ratio (AR).

22 Features have been selected from the NSLKDD dataset and for classification, the J48 classifier has been used. Genetic Principal Component (GPA) [27] approach has been used to select optimal features from the KDDCUP99 dataset with SVM classifier for intrusion detection. In order to develop an intelligent IDS using the NSLKDD dataset, Manekar et al. [28] used parameter turning using Particle Swarm Optimization (PSO) with SVM classifier. Another variant of PSO is the intrusion feature selection algorithm (IFSA) based PSO [29, 30]. Which represents velocity and position in intervals compare to a single numeric value. The technique has been used on the KDD99 dataset, while random based PSO has also been used for intrusion detection [31]. PSO can improve the performance of the Multiple Criteria Linear Programming (MCLP) classifier [32]. PSO provides a selection of optimal features for various datasets such as KDDCUP99 [33]. We have investigated various feature selection techniques and performed an analysis of 
the available systems that can classify a packet into normal or anomaly classes automatically. We have examined the available literature using the following criteria,as shown in table 1.

TAB LE 1 Survey on feature selection and classification techniques

\begin{tabular}{|c|c|c|c|c|c|}
\hline Author & Year & Feature selection & Features & Classifier & Dataset \\
\hline Heba et.al.[25] & 2010 & PCA & 23 & SVM & NSLKDD \\
\hline Mukherjee et.al.[34] & 2012 & FVBRM & 24 & Naïve Bayes & NSLKDD \\
\hline \multirow{4}{*}{ H.Chae et.al.[26] } & \multirow{4}{*}{2013} & AR & 22 & \multirow{4}{*}{ J48 } & \multirow{4}{*}{ NSLKDD } \\
\hline & & CFS & 25 & & \\
\hline & & IG & 23 & & \\
\hline & & GR & 19 & & \\
\hline Tesfahun et.al.[35] & 2013 & IG & 22 & Random Forest & NSLKDD \\
\hline \multirow{5}{*}{ Eesa et.al. [27] } & \multirow{5}{*}{2014} & RAW & RAW 38 & \multirow{5}{*}{ SVM } & \multirow{5}{*}{ KDD99 } \\
\hline & & PCA & PCA 38 & & \\
\hline & & PCA & PCA 22 & & \\
\hline & & \multirow{2}{*}{ GPC } & GPC 12 & & \\
\hline & & & GPC 10 & & \\
\hline V.Manekar et.al.[28] & 2014 & PSO & - & SVM(RBF) & NSLKDD \\
\hline Shrivas et.al.[36] & 2014 & GR & 35 & ANN+Bayesian Net & NSLKDD \\
\hline Patel et.al.[31] & 2015 & PSO & - & - & NSLKDD \\
\hline Ahmad et.al.[37] & 2015 & $P C A+P S O$ & 8 & MNN & NSLKDD \\
\hline Eesa et.al.[38] & 2015 & CFA & 5 & Decision Tree & KDD99 \\
\hline \multirow[t]{2}{*}{ K.Rai et al [39] } & 2016 & Information Gain & 16 & DTS & NSLKDD \\
\hline & & & & & KDD99 \\
\hline \multirow[t]{2}{*}{ Bamakan et al.[40] } & 2016 & FMIFS & $19,18,4$ & LSSVM & NSLKDD \\
\hline & & & & & Kyoto 2006 \\
\hline Bamakan et al.[41] & 2016 & TVCPSO & 17 & SVM & NSLKDD \\
\hline Thaseen et.al.[42] & 2017 & Chi & 31 & SVM & NSLKDD \\
\hline Syarif et.al.[33] & 2017 & PSO & 25 & KNN & KDD99 \\
\hline Pajouh et.al.[43] & 2018 & - & 41 & Deep Learning & NSLKDD \\
\hline Shone et.al.[44] & 2018 & - & 41 & RNN & NSLKDD \\
\hline Naseer et.al.[45] & 2018 & - & 41 & LSTM & NSLKDD \\
\hline Sakr et.al.[46] & 2019 & $\mathrm{BPSO}+\mathrm{SPSO}+\mathrm{SVM}$ & 23 & SVM & NSLKDD \\
\hline Woo et.al.[47] & 2019 & Correlation Method & 40 & Neural Network & NSLKDD \\
\hline
\end{tabular}


From Table 1, we can conclude that though, PCA provides an optimal feature set, but it compromises the training efficiency [48]. The problem with information gain and Gini-index is it give biased results for non-numeric values [49]. Similarly with genetic algorithm and fuzzy logic does not provide surety for optimal solutions [50]. Therefore, more robust solutions are required, which not only give optimal solutions but also have a fast convergence rate, unlike the genetic algorithm, which has a slow convergence rate, also depends upon the population used [51]. That's why we used weighted PSO for feature optimization to make the system more robust. PSO will automatically provide a set of optimal features regardless of the dataset. The above mention feature selection methods either improved detection rate, accuracy, or false alarm rate not all the measures at the same time and on different datasets. These feature selection methods are data-dependent. Therefore, a more optimal way is required, which can solve the above mention problems and perform well regardless of the dataset. For this reason we have proposed, weighted PSO in this research, which achieved promising results compare to other studies.

\section{3 | PROPOSED MODEL}

This research proposes an artificial intelligence (AI) base solution for the data-driven security part of the smart grid system by using the optimal features subset and Al models. The objective of this research is to propose a machine learning model which detects network traffic packets quickly and accurately while achieving a low False Alarm Rate (FAR) and high Detection Rate (DR). To achieve this objective optimal feature selection is very important to be used. In this research, the PSO search algorithm is implemented to select the best features from a given subset of features. The datasets used in this research are NSLKDD and KDD99. For both KDD99 and NSLKDD datasets, we perform binary classification, i.e., anomaly or normal, as well as multiclass classification to predict attack categories, such as Denial of Service (DoS), R2L, U2R, Probe and Normal class. After a successful classification of the attacks, we do further classification to handle the exact name of the anomaly. The proposed model consists of six phases. The 1 st is data reading, in the data reading phase, we read KDD99 and NSLKDD datasets one by one. The $2 n d$ is data preprocessing, in the preprocessing step, we replace missing values by mean, remove the outliers in data if any, after that data normalization is performed to scale the data. After completing the data normalization, then we performed data encoding to convert non-numeric values into numeric values. The last stage of data preprocessing is the optimal feature selection, which is performed using PSO. The complete working of PSO is discussed in the next section. The $3 r d$ is passing optimal features to machine learning selected models. In the 4 th phase, we trained different models by passing $70 \%$ data and labels to the model. Testing is performed on $30 \%$ of the data. 5 th phase phase is the experiment phase and 6th phase phase is evaluation.Figure 3 represent the proposed model.

\section{1 | Datasets}

\subsection{1 | KDD99 dataset}

KDD99 is one of the most famous datasets used in the field of network security for IDS. KDD99 is a derived version of the 1998 DARPA. It is developed in the MIT research lab and is used by IDS designers as a benchmark to evaluate various methodologies and techniques [52, 53]. KDD99 has 4,900,000 rows and 41 attributes having binary labels and 22 network attacks are listed in the KDD99 dataset. Class labels consist of 4 major attacks like DoS, Probe, U2R, R2L and Normal class. 


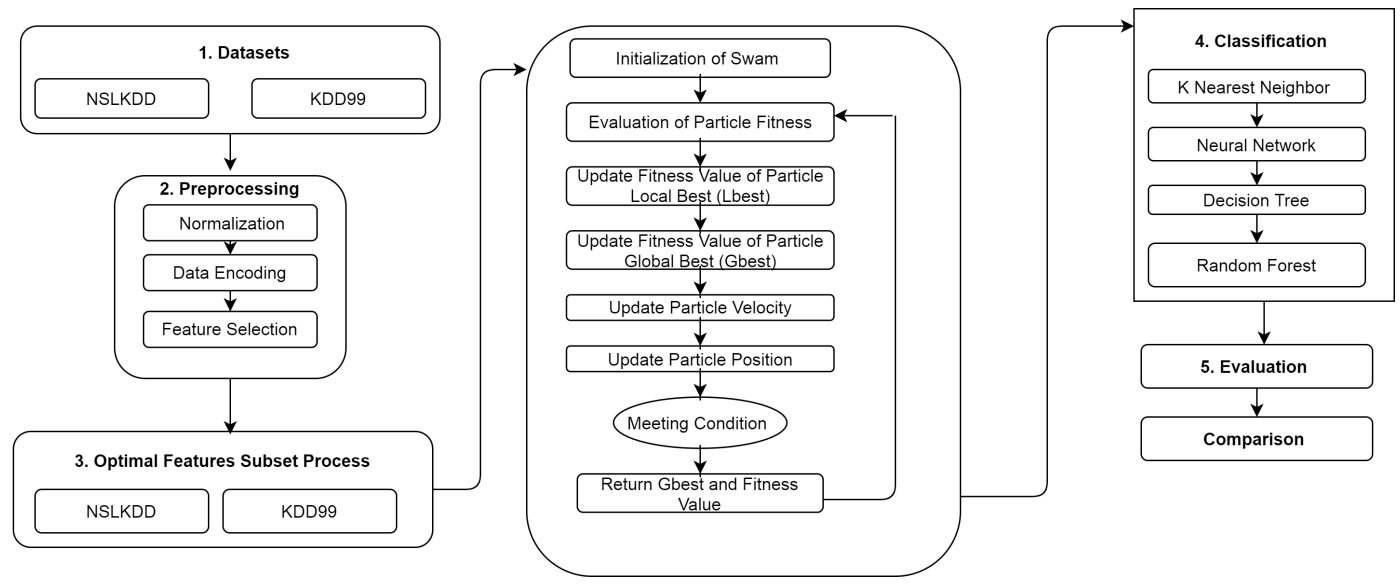

FIGURE 3 Proposed methodology

TAB LE 2 KDD99 dataset normal and anomaly packets

\begin{tabular}{ll}
\hline Normal packets & 97277 \\
Anomaly packets & 396731 \\
Total size & 494008 \\
\hline
\end{tabular}

Table 2 represents the total number of normal and anomaly packets contain the KDD99 dataset used in this research. 97277 and 396731 packets are used for anomaly and normal class to develop ensemble machine learning classifiers upon which training and testing can be performed. 70\% KDD99 dataset is used for training and validation purpose and the rest of the $30 \%$ dataset is used for testing and validation, respectively.

\subsection{2 | NSLKDD dataset}

NSLKDD is an updated copy of the KDD99 dataset. NSLKDD does not have any duplicate Values, which is in the KDD99 dataset. NSLKDD also does not have any inconsistent values. NSLKDD contains 148517 instances and 41 features for training and testing purposes overall.

TAB LE 3 NSLKDD dataset normal and anomaly packets

$\begin{array}{ll}\text { Normal packets } & 77054 \\ \text { Anomaly packets } & 71215 \\ \text { Total size } & 148269\end{array}$

Table 3 represents the total number of normal and anomaly packets contain the NSLKDD dataset used in this research. The total number of an anomaly and normal packets used to train and test machine learning models are 71215 and 77054, respectively. 70\% KDD99 dataset is used for training and the rest of the $30 \%$ dataset is used for testing and validation, respectively. 
TABLE 4 NSLKDD dataset training and testing packets

\begin{tabular}{ll}
\hline Training data size & 103789 \\
Testing data size & 44481 \\
\hline
\end{tabular}

Table 4 represents the total number of an anomaly and normal packets used to train and test machine learning models are 103789 and 44481, respectively. Table 5 represents the number of features in both the datasets.

TAB LE 5 Total number of features in KDD99 and NSLKDD datasets

\begin{tabular}{|c|c|c|c|}
\hline Feature Name & Feature Type & Feature Name & Feature Type \\
\hline Duration & Number & Protocol type & Non-Numeric \\
\hline Service & Non-Numeric & Flag & Non-Numeric \\
\hline Src bytes & Number & Destination bytes & Number \\
\hline Land & Non-Numeric & Wrong fragt & Number \\
\hline Urgent & Number & Hot & Number \\
\hline Num of failed logins & Number & logged in & Non-Numeric \\
\hline Num access files & Number & Root shell & Number \\
\hline Su_Attemped & Number & Number root & Number \\
\hline Number of file creations & Number & Number shells & Number \\
\hline Number access files & Number & Number outbound commands & Number \\
\hline Is host login & Non-Numeric & Is guest login & Non-Numeric \\
\hline Count & Number & Service Count & Number \\
\hline Serror rate & Number & Service Error rate & Number \\
\hline Rerror rate & Number & Service error rate & Number \\
\hline Same service rate & Number & Different service rate & Number \\
\hline Service different host rate & Number & Dst_host_count & Number \\
\hline Dst_host_srv_count & Number & Dst_host_same_srv_rate & Number \\
\hline Dst_host_diff_srv_rate & Number & Dst_host_same_src_port_rate & Number \\
\hline Dst_host_srv_diff_host_rate & Number & Dst_host_serror_rate & Number \\
\hline Dst_host_srv_serror_rate & Number & Dst_host_rerror_rate & Number \\
\hline Dst_host_srv_rerror_rate & Number & Class label type & Non-Numeric \\
\hline
\end{tabular}




\section{2 | Pre-processing}

\subsubsection{Normalization:}

After selection of dataset, data cleaning operations are performed on datasets to remove noise from dataset and normalize the features. For normalization different techniques are used but in this research min-max normalization approach is used which is better in terms of scaling and solve outliers' issues with z-score normalization Min-max scaling normalizes values in the range of $[0,1]$. Equation for min-max normalization is given below.

$$
Z_{i}=\frac{Y_{i}-\min (Y)}{\max (Y)-\min (Y)}
$$

From equation 1, $Y=(Y 1, Y 2, Y 3 \ldots Y n)$ are the number of features while $Y_{i}$ is the feature which we want to normalize and $Z_{i}$ are normalized features. By doing this now all features have same weights and all features are in one scope.

\subsection{2 | Data encoding}

Before data encoding, we remove duplicate and inconsistent values from the datasets. Then the nominal attributes are converted to numeric, the reason for that machine learning algorithms back end calculations are done on numeric values not nominal values. So this step is done before passing data to the proposed model.

\section{\begin{tabular}{l|l} 
3.2.3 & Feature selection
\end{tabular}}

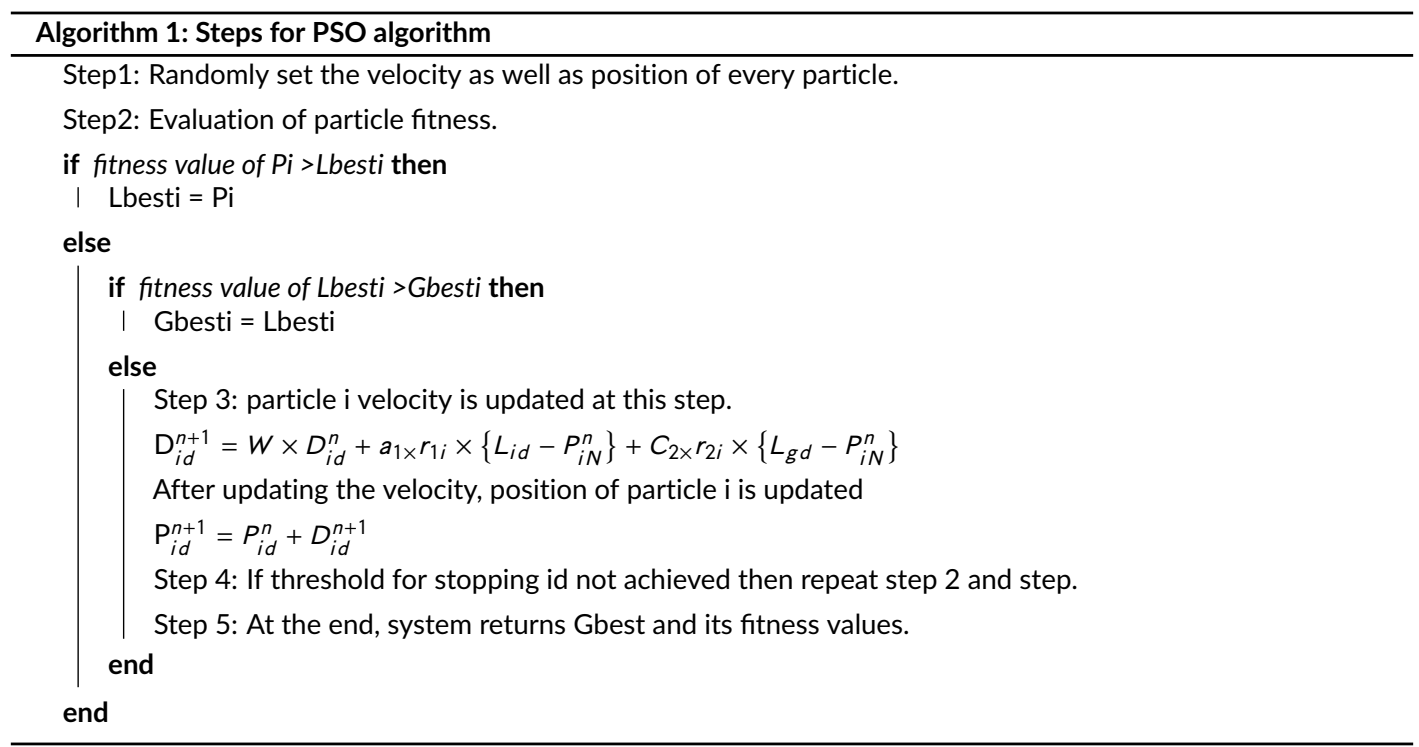

After feature normalization next important step is the feature optimization. Optimal features not only improve accuracy, but also improve detection rate and false alarm rate. The main focus of feature optimization is to find such feature subsets that can work with different classifiers to produce better results. In this research, we use PSO search method for feature selection. Eberhart and Kennedy [54] in 1995 inspired from fish and birds flock movement behavior and proposed PSO which is generally an optimization algorithm. To solve non-smooth global problems PSO 
is considered one of the powerful technique [51].Convergence rate of PSO is also very high and it gives optimal solution in less amount of time [55]. Genetic algorithms are also used for optimal feature selection which produce good detection rate but the issue with genetic algorithms is that their convergence rate is very slow and may become worse if subjects of the population are also used [56]. The swarm particles are randomly initialized and then passed to search arena, by changing the value for velocity and for position of particle we can get optimal features subset. The present position and its velocity are expressed in (2) and (3).

$$
P_{i}=\left\{P_{i 1}, P_{i 2}, P_{i 3}, P_{i 4}, P_{i 5}, P_{i 6} \ldots \ldots P_{i N}\right\}
$$

Where the dimension of principal search space is represented by $\mathrm{N}$.

$$
D_{j}=\left\{D_{j 1} D_{j 2} D_{j 3} D_{j 4} D_{j 5} D_{j 6} \ldots \ldots D_{j N}\right\}
$$

Until we get the optimal values algorithm keep updating values for velocity as well as for position. As soon as we get the optimal features, the algorithm stops.

\section{3 | Selected optimal features for NSLKDD and KDD99 datasets}

TABLE 6 NSLKDD selected optimal attributes

\begin{tabular}{lll}
\hline S. No & Feature Name & Data Type \\
\hline 1 & Service & Nominal \\
2 & Destination bytes & Numeric \\
3 & Logged-in & Numeric \\
4 & Count & Numeric \\
5 & Srv-diff-host-rate & Numeric \\
6 & Dst-host-count & Numeric \\
7 & Labels & Nominal \\
\hline
\end{tabular}

TAB LE 7 KDD99 selected optimal attributes

\begin{tabular}{lll}
\hline S. No & Feature Name & Data Type \\
\hline 1 & Service & Numeric \\
2 & Destination bytes & Numeric \\
3 & Logged-in & Numeric \\
4 & Count & Numeric \\
5 & Srv_diff_host_rate & Numeric \\
6 & Dst-host-count & Numeric \\
7 & Dst-host-srv-diff-host-rate & Numeric \\
8 & Labels & Nominal \\
\hline
\end{tabular}


Table 6 and table 7 represents the optimal features selected form NSLKDD and KDD99 datasets.

\section{4 | Classifiers}

\subsection{1 | K-Nearest Neighbor}

K-Nearest Neighbor Classifier (KNN) uses similarity measures to predict new data points. The reason for using the KNN algorithm in this research is that it depends upon the features' similarity. To achieve optimal results, the selection of the right value of $\mathrm{K}$ is significant. The value of $\mathrm{K}$ is the number of nearest neighbors that are considered in the classification of a vector. In this research, we select $K=5$, leaf-size=30 and Minkowski metric is used along weights are uniformed. Equations for $\mathrm{KNN}$ is given below

$$
\begin{aligned}
& \text { Eculidean equation }=\sqrt{\sum_{i=1}^{k}(X i-Y i)^{2}} \\
& \text { Manhattan equation }=\sum_{i=1}^{k}|X i-Y i| \\
& \text { Minkowski }=\left(\sum_{i=1}^{k}(|X-Y i|)^{q}\right)^{1 / q}
\end{aligned}
$$

\subsection{2 | Neural Network (NN)}

An NN is a data processing paradigm that is motivated by the biological sensory system. Such as the human brain. The neural network is also widely used in IDS and it is represented in figure 4. Given an input node $X_{a}$, the output of the hidden node $O_{b}$ is given as:

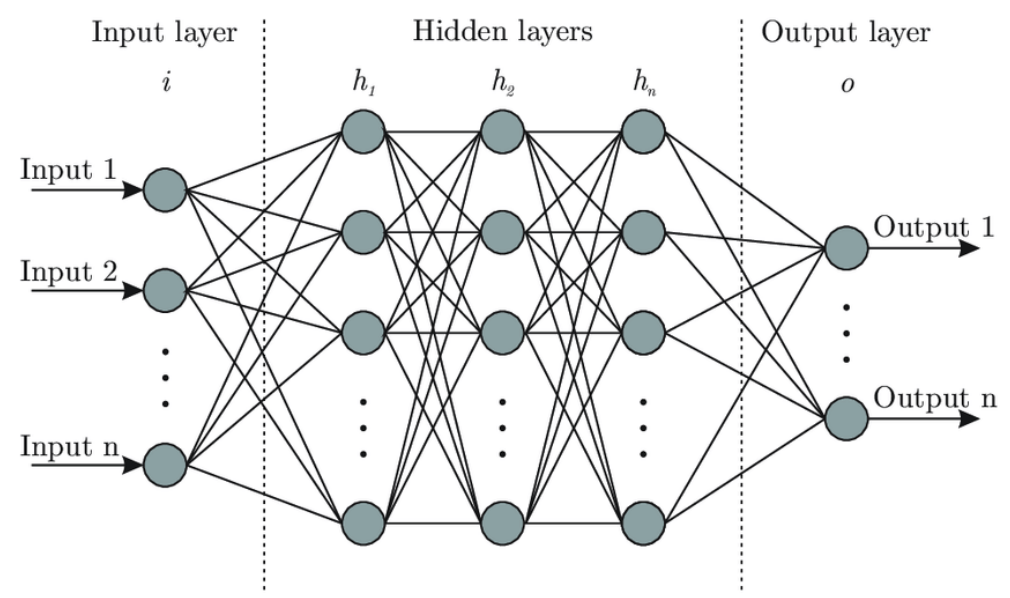

FIG URE 4 Neural Network structure [57] 


$$
O_{b}=\phi_{1}+\left(\sum_{a=1}^{n} U_{a b}+\theta_{b}\right)
$$

where $w a, b$ represents the weight between the $a t h$ input and $j_{t h}$ hidden node, and $\theta_{j}$ represents the bias value. Whereas, output will be given

$$
\text { output }=\phi_{2}+\left(\sum_{b=1}^{n} U_{b k}+\theta_{k}\right)
$$

The mapping of inputs to outputs is an iterative process, where in each iteration weights Ua,b are updated. One of the commonly used algorithm is Back Propagation algorithm which updates the weights using:

$$
U_{b a}(t+1)=U_{b a}(t)-\epsilon \frac{\partial E f}{U_{b a}}
$$

The NN is mostly used to solve complex problems and it consists of the input layer, weighted (hidden layers) and output layers. Weights are assigned to each layer in the neural network system. The activation function is also used in the neural network. The NN Model is represented in figure 4. A neural network consists of 60 hidden layers with an activation function of relu, and alpha size is 0.0001 . We kept the batch size constant. Max-Iter is 200 and randomness is true.

\subsection{3 | Decision Tree}

Another algorithm used in recent anomaly-based IDS research is the Decision Tree (DT), this is the same as any tree structure consisting of edges, nodes, leaves etc. A feature and threshold is typically applied to a node and the data is split down the tree, where for example if the data is below a threshold it goes left and above a threshold goes right, until it ends up in a final cluster or class [18]. One DT method is ID3 algorithm that quantifies information by using entropy. Equations for entropy is given below

$$
\text { Entropy: } H\left(p_{1}, p_{2}, \ldots p_{2}\right)=\sum_{i=1}^{s}\left(p_{i} \log \left(1 / p_{i}\right)\right.
$$

Where $(\mathrm{p} 1, \mathrm{p} 2, \ldots \mathrm{ps})$ represents the probabilties of the class labels.

$$
\operatorname{Gain}(D, S)=H(D) \sum_{i=1}^{s} p\left(D_{i}\right) H\left(D_{i}\right)
$$

Another decision tree method is called the C4.5. Decision tree [58, 59] has the ability to process large amounts of data efficiently is used to sort data into groups so that a Support Vector Machine (SVM) can classify the smaller subsets of information. In [60] author proposed a similar method however an SVM is placed on each edge in the DT. We performed splitting using gini-index, max-depth=none, min-samples-split=2, min-samples-leaf=1, class-weights=none, random-state=none, $\min$-impurity-decrease $=0.0$ and $\min$-impurity-split=none. 


\subsection{4 | Random Forest}

Random Forest classifier plays a significant part in IDS. It is a combination of multiple decision trees and random forest combine all the decision trees to get prediction sharpened and get more accurate results. The best thing about the random forest is that it can be used for both regression and classification. The random forest also tells us about the importance of the features that will help in deciding which features should be kept and which ones should be dropped from the dataset.

\subsection{Evaluation metrics}

Various performance metrics are used to evaluate the proposed solution, including precision, recall, F1-Measure [61], False Alarm Rate (FAR), Detection Rate (DR) and Accuracy. Above mention performance metrics base on True Positive (TP), False Positive (FP), False Negative (FN) and True Negative (TN).

False Alarm Rate (FAR) is a combination of total instances that are normal but classify as attack class and truly classify attack class.

$$
F A R=\frac{F P}{F P+T N}
$$

Accuracy [62] is used to measure how many instances are correctly classified as normal and attacks classes. Accuracy is achieved by summing correctly classify instances with dividing the total instances represented in equation 13.

$$
\text { Accuracy }=\frac{T P+T N}{T P+F P+F N+T N}
$$

Detection Rate (DR) provides information about the attacks detected correctly divided by the total number of attacks in the dataset.

$$
D R=\frac{T P}{T P+F N}
$$

Precision's objective is to evaluate the True Positive (TP) entities in relation to False Positive (FP) entities.

$$
\text { Precision }=\frac{T P}{T P+F P}
$$

The purpose of recall is to evaluate True Positive (TP) entities in relation to (FN) False Negative entities that are not at all categorized. The mathematical form of recall is mentioned in equation (16).

$$
\text { Recall }=\frac{T P}{T P+F N}
$$


Sometimes performance assessment may not be good with accuracy and recall, For instance, if one mining algorithm has low recall but high precision that another algorithm is needed. Then there is the question of which algorithm is better. This problem is solved by using F1-score that gives an average recall and precision. F1-score can be calculated as shown in equation (17).

$$
F 1-\text { score }=\frac{2 * \text { Precision } * \text { Recall }}{\text { Precision }+ \text { Recall }}
$$

\section{4 | EXPERIMENT RESULTS}

In this section experiment results of KDD99 and NSLKDD are mentioned. All these experiments are performed on google colab. System specification core 13 system with 8 GB RAM and $2.7 \mathrm{GHz}$ processor is used.

TABLE 8 Classification report for KDD99

\begin{tabular}{|c|c|c|c|c|}
\hline Model Name & Class & Precision \% & Recall \% & F1-score \% \\
\hline \multirow[t]{2}{*}{$\mathrm{PSO}+\mathrm{KNN}$} & Normal & 98.8 & 97.6 & 98.2 \\
\hline & Attack & 99.4 & 99.7 & 99.6 \\
\hline \multirow[t]{2}{*}{ PSO + Neural Network } & Normal & 95.4 & 99.6 & 97.5 \\
\hline & Attack & 99.9 & 98.8 & 99.4 \\
\hline \multirow[t]{2}{*}{ PSO + Decision Tree } & Normal & 98.5 & 99.2 & 98.8 \\
\hline & Attack & 99.8 & 99.6 & 99.7 \\
\hline \multirow[t]{2}{*}{ PSO + Random Forest } & Normal & 98.5 & 99.3 & 98.9 \\
\hline & Attack & 99.8 & 99.6 & 99.7 \\
\hline
\end{tabular}

From Table 8, we can conclude that precision, recall and f1-score for $\mathrm{KNN}$, normal class is $98.89 \%, 97.60 \%$, $98.20 \%$, respectively. Similarly, for an anomaly class, precision is $99.40 \%$, the recall is $99.70 \%$ and the f1-score is 99.60\%, respectively. Random forest precision, recall and f1-score for the normal class will give us $98.50 \%, 99.30 \%, 98.90 \%$, respectively. Precision, recall and f1-score for attack class are 99.80\%, 99.60\%,99.70\%. For decision tree and neural network, precision scores for the normal class are $98.50 \%, 95.40 \%$, respectively. Similarly, recall and f1-scores are $\mathbf{9 9 . 3 0 \%}$ and $\mathbf{9 8 . 4 0 \%}$ on average for a normal class. Precision recall and f1-scores on average for an attack class using decision tree and neural network are 97\%, 99.60\% 99.50\% respectively depicted in the figure 5 . 


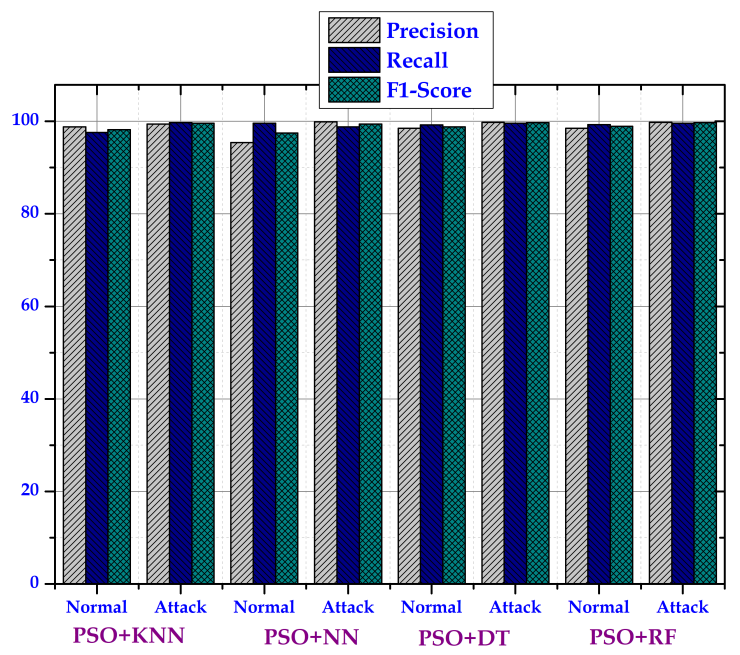

FIGURE 5 Classification report for KDD99 datasets

TABLE 9 FAR, DR and Accuracy comparison report

\begin{tabular}{lll}
\hline Model Name & KDD99 (FAR \%) & NSLKDD (FAR \%) \\
\hline PSO + KNN & 2.40 & 0.17 \\
PSO + Neural Network & 0.50 & 3.13 \\
PSO + Decision Tree & 0.80 & 0.14 \\
PSO + Random Forest & 0.60 & 0.08 \\
\hline
\end{tabular}

Table 9 and figure 6 depicts that the KNN classifier with KDD99 dataset achieved 2.4\% FAR which is high compare to other classifiers, decision tree and random forest achieved $0.8 \%$ and $0.6 \%$ FAR respectively. For KDD99 neural network outperformed other classifiers in terms of FAR and it achieved 0.5\% FAR. The reason for this is neural network performs well on large dataset and KDD99 dataset has more data compare to NSLKDD dataset. Similarly random forest achieved promising results for FAR using NSLKDD dataset. FAR for random forest is $0.08 \%$, since random forest is ensemble classifier and it is the combination of multiple decision tree that's why it achieved promising results compare to other classifiers like decision tree, KNN and NN.

From table 10 and table 11 we can conclude that using the KNN classier with KDD99 dataset, 118779 packets are identified as an attack, while only 337 packets are misclassified out of 119116 packets. For normal class out of 29090 packets, 28390 packets are detected correctly and 700 packets are identified incorrectly with the accuracy of $97.60 \%$ for normal class and $99.70 \%$ for attack class, respectively. The detection rate for the knn classifiers is $99.70 \%$. True positive for random forest and decisions tree are 118672 and 118680 , respectively. The true negative for the random forest is 28902 . Similarly, for the decision tree the true negative is 28850 . False positive and false negative scores for the random forest is 188 and 444, respectively. For the decision tree overall, 676 packets are misclassified. The detection rate for both the random forest and the decision tree is $99.60 \%$, respectively. The neural network also 


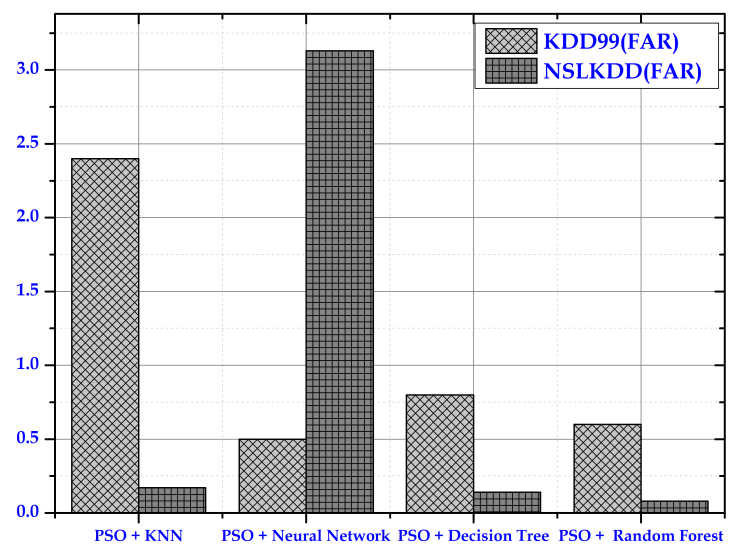

FIGURE 6 FAR for KDD99 and NSLKDD datasets

achieved promising results for true positive and for true negative with the detection rate of $99.20 \% .118161$ packets are correctly detected as an attack with an accuracy of $99.20 \%$, while 28927 packets are correctly identified normal packets with an accuracy of $99.40 \% .95$ packets are misclassified for attack class and 163 packets for the normal class using a neural network.Figure 7 represents accuracy and detection rate for both datasets.

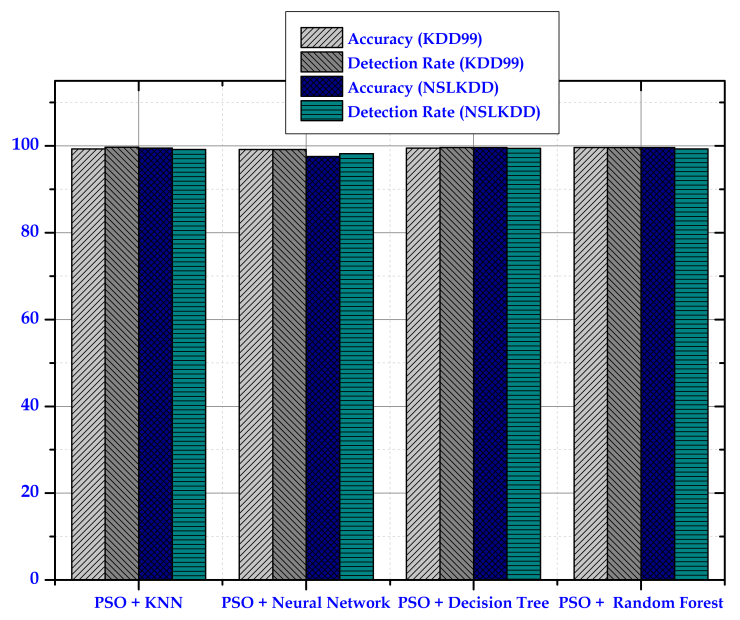

FIGURE 7 Accuracy and DR for both datasets. 
TABLE 10 Confusion matrix for KDD99

\begin{tabular}{lllll}
\hline Model Name & TP & FN & FP & TN \\
\hline PSO+ KNN & 118779 & 337 & 700 & 28390 \\
PSO+ Neural Network & 118161 & 95 & 163 & 28927 \\
PSO+ Decision Tree & 118680 & 436 & 240 & 28850 \\
PSO+ Random Forest & 118672 & 444 & 188 & 28902 \\
\hline
\end{tabular}

TABLE 11 Accuracy and DR for both datasets.

\begin{tabular}{llllll}
\hline \multirow{2}{*}{ Model Name } & \multicolumn{2}{c}{ KDD99 } & \multicolumn{2}{c}{ NSLKDD } \\
\cline { 2 - 5 } & Accuracy \% & DR \% & Accuracy \% & DR \% \\
\hline PSO+KNN & 99.3 & 99.7 & 99.51 & 99.17 \\
PSO+NN & 99.2 & 99.2 & 97.54 & 98.18 \\
PSO+DT & 99.5 & 99.6 & 99.64 & 99.41 \\
PSO+RF & 99.6 & 99.6 & 99.65 & 99.3 \\
\hline
\end{tabular}

TABLE 12 Confusion matrix for NSLKDD

\begin{tabular}{lllll}
\hline Model Name & TP & FN & FP & TN \\
\hline PSO+ KNN & 21255 & 176 & 41 & 23083 \\
PSO+ Neural Network & 21041 & 390 & 703 & 22421 \\
PSO+ Decision Tree & 21306 & 125 & 34 & 23090 \\
PSO+ Random Forest & 21295 & 136 & 20 & 23104 \\
\hline
\end{tabular}

Table 11 and table 12 represents that the random forest with PSO achieved $99.65 \%$ accuracy and $99.30 \%$ detection rate, respectively. Precision, recall and f1-scores are $99.40 \%, 99.90 \%, 99.70 \%$ respectively for a normal class. Similarly, for an anomaly class, we achieved $99.90 \%$ precision, $99.40 \%$ recall and $99.60 \%$ f1-score, respectively. KNN model gained $99.51 \%$ accuracy overall, for normal class accuracy is $99.8 \%$, while for an attack class, accuracy is 99.20\%. Decision tree detected 21307 packets correctly as anomaly out of 21431 with the accuracy of $99.40 \%$ and out of 23124 normal packets, 23093 packets correctly identified as normal traffic with the accuracy of $99.90 \%$. For an attack class decision tree achieved $99.80 \%$ precision, recall is $99.40 \%$ and $99.70 \% \mathrm{f} 1$-score, similarly for normal class precision is $\mathbf{9 9 . 5 0 \%}$ while recall is $99.90 \%$ and $\mathrm{f} 1$-score is $99.70 \%$. Using a multilayer perceptron, we achieved 99.50\% accuracy for normal class and $97.90 \%$ accuracy for anomaly class. $98.5 \%$ overall accuracy is achieved in [42]. Similarly, in [61] they got $97.87 \%$ overall accuracy. We gained a $98.18 \%$ detection rate while the false alarm rate is around $3.13 \%$ using a multilayer perceptron. MLP results are a little low compare to knn, decision tree and random forest, the reason for this is a neural network performs well when class is balance and when we have a large amount of data for both training and testing. For a normal class preicion, recall and f1-score is $95.10 \%, 99.90 \%$ and $97.40 \%$ 
respectively using multilayer perceptron classifier and NSLKDD dataset. Similarly, for an anomaly class, precision, recall and f1-score is $99.90 \%, 94.50 \%, 97.10 \%$, respectively, depicated in table 13 and figure 8 .

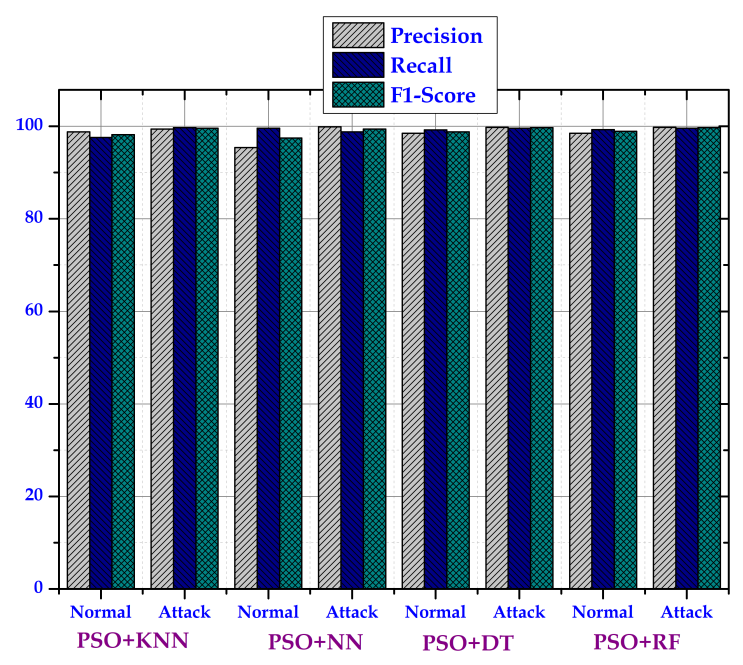

FIGURE 8 Classification report for NSLKDD dataset.

TAB LE 13 Classification report for NSLKDD

\begin{tabular}{lllll}
\hline Model Name & Class & Precision \% & Recall \% & F1-score \% \\
\hline PSO + KNN & Normal & 99.2 & 99.8 & 99.5 \\
& Attack & 99.8 & 99.2 & 99.5 \\
PSO + Neural Network & Normal & 95.1 & 99.9 & 97.4 \\
& Attack & 99.9 & 94.5 & 97.1 \\
PSO + Decision Tree & Normal & 99.5 & 99.9 & 99.7 \\
& Attack & 99.8 & 99.4 & 99.6 \\
PSO + Random Forest & Normal & 99.4 & 99.9 & 99.7 \\
& Attack & 99.9 & 99.4 & 99.6 \\
\hline
\end{tabular}

\section{1 | KDD99 Multi Class Classification Experimental Results}

Table 14 and figure 9 depict that normal class achieved $98.30 \%$ precision, $96.10 \%$ recall and $97.10 \%$ F1-Measure, respectively. TP and FP rate is $96.10 \%$ and $0.4 \%$ respectively. Smurf and Warezclient achieved a $100 \%$ detection rate, respectively. Similarly, for Warezclient and Smurf attack has 0\% and 0.3\% FP rate, respectively. Recall for both Warezclient and Smurf attacks is $100 \%$, respectively, while f1-score is above $99 \%$ on average for both the attacks, respectively. Precision for Warezclient is $99.30 \%$ and Smurf precision is $98.9 \%$, respectively, for Portsweep DR and 
recall is $89.20 \%$, respectively. FP rate for Portsweep is high compare to other attacks using a decision tree, which is around $1.8 \%$. Precision and F1-Measure scores are $77.20 \%$ and $82.80 \%$ respectively for Portsweep. On average, precision, recall, F1-Measure and TP rate scores for Ipsweep are $98.50 \%$ and the FP rate is $0.2 \%$, respectively. Saran, Nmap, Back, Teardrop and Neptune also performed well and achieved, on average, 93\% precision, recall and F1Measure, respectively.

TAB LE 14 Classification report for Decision Tree

\begin{tabular}{llllll}
\hline Class & TP Rate $\%$ & FP Rate \% & Precision \% & Recall \% & F1-score \% \\
\hline saran & 84.7 & 0.3 & 97.3 & 84.7 & 90.6 \\
portsweep & 89.2 & 1.8 & 77.3 & 89.2 & 82.8 \\
ipsweep & 99.1 & 0.2 & 96.7 & 99.1 & 97.9 \\
nmap & 41.2 & 0 & 96.6 & 41.2 & 57.7 \\
back & 97.9 & 0.1 & 98.9 & 97.9 & 98.4 \\
teardrop & 86.1 & 1.5 & 76.6 & 86.1 & 81.1 \\
warezclient & 100 & 0 & 99.3 & 100 & 99.7 \\
neptune & 95.6 & 1.7 & 92.8 & 95.6 & 94.2 \\
smurf & 100 & 0.3 & 98.9 & 100 & 99.4 \\
normal & 96.1 & 0.4 & 98.3 & 96.1 & 97.1 \\
\hline
\end{tabular}

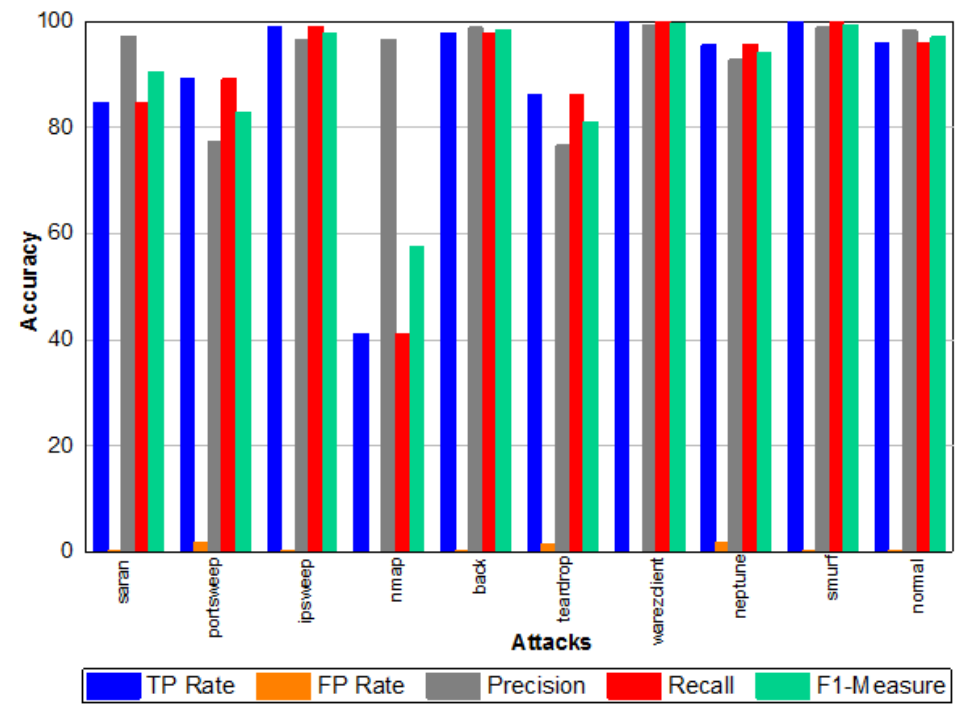

FIGURE 9 Classification report for Decision Tree 
TAB LE 15 Classification report for Random Forest

\begin{tabular}{llllll}
\hline Class & TP Rate $\%$ & FP Rate \% & Precision \% & Recall \% & F1-score \% \\
\hline saran & 85.1 & 0.5 & 95.3 & 85.1 & 89.9 \\
portsweep & 89.5 & 1.9 & 76.5 & 89.5 & 82.5 \\
ipsweep & 99.1 & 0 & 99.4 & 99.1 & 99.2 \\
nmap & 42.6 & 0 & 96.7 & 42.6 & 59.2 \\
back & 97.9 & 0.1 & 99.2 & 97.9 & 98.5 \\
teardrop & 81.1 & 1.6 & 73.9 & 81.1 & 77.3 \\
warezclient & 100 & 0 & 99.3 & 100 & 99.7 \\
neptune & 96.1 & 1.7 & 92.6 & 96.1 & 94.3 \\
smurf & 100 & 0 & 99.9 & 100 & 99.9 \\
normal & 96.3 & 0.4 & 98.2 & 96.3 & 97.2 \\
\hline
\end{tabular}

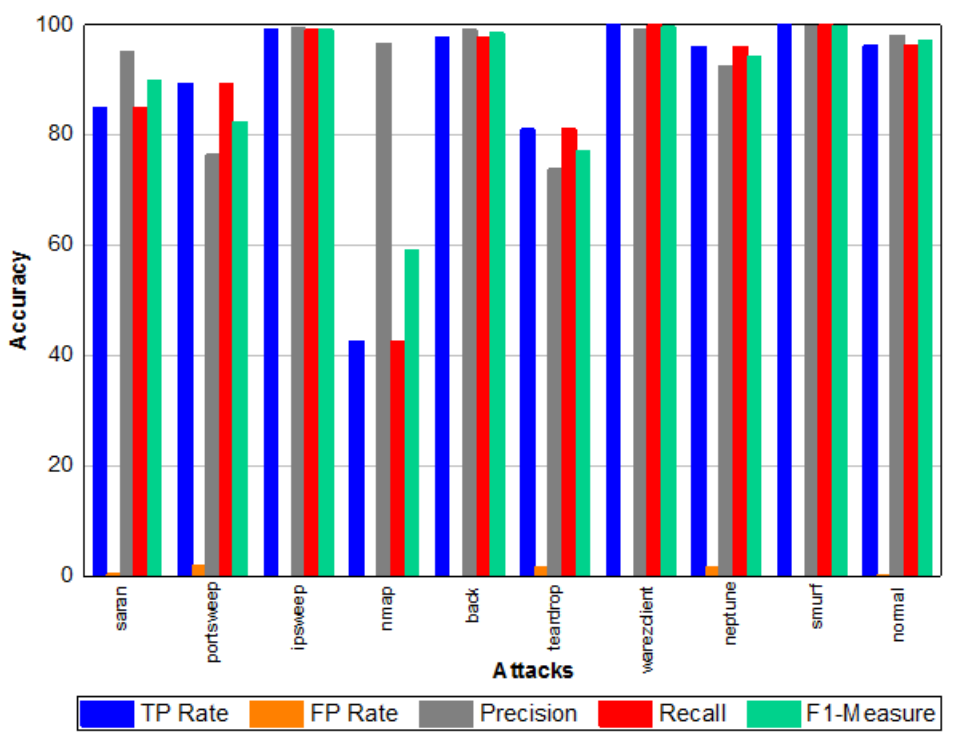

FIGURE 10 Classification report for Random Forest

From table 15 and figure 10, we can conclude that the FR rate for Ipsweep, Nmap, Warezclient and Smurf is $0 \%$, respectively, which is promising. Similarly, the DR rate for those attacks is $99.10,42.60 \%, 100 \%$, respectively. Saran, Portsweep, Back, Teardrop and Neptune achieved 0.5\%, 1.9\%, 0.1\%, 1.6\% , 1.7\% FR rate respectively.The DR rate for those attack is $85.10 \%, 89.50 \%, 97.90 \%, 81.10 \%, 96.10 \%$ respectively.Precision, recall and F1-Measure for all attacks on average are $92.50 \%, 87.93 \%, 88.88 \%$, respectively. For normal class precision, recall and F1-Measure 
is $98.20 \%, 96.30 \%, 97.20 \%$, respectively.TP and FP for normal class is $96.30 \%$ and $0.4 \%$, respectively.

TAB LE 16 Classification report for K Nearest Neighbour

\begin{tabular}{llllll}
\hline Class & TP Rate $\%$ & FP Rate \% & Precision \% & Recall \% & F1-score \% \\
\hline saran & 84.9 & 0.5 & 94.6 & 84.9 & 89.5 \\
portsweep & 90.5 & 1.9 & 76 & 90.5 & 82.6 \\
ipsweep & 99.1 & 0 & 100 & 99.1 & 99.5 \\
nmap & 42.6 & 0 & 96.7 & 42.6 & 59.2 \\
back & 97.6 & 0.2 & 98.1 & 97.6 & 97.9 \\
teardrop & 83 & 1.7 & 73.9 & 83 & 78.2 \\
warezclient & 100 & 0 & 100 & 100 & 100 \\
neptune & 95.4 & 1.6 & 93.3 & 95.4 & 94.3 \\
smurf & 100 & 0 & 100 & 100 & 100 \\
normal & 95.8 & 0.5 & 97.7 & 95.8 & 96.8 \\
\hline
\end{tabular}

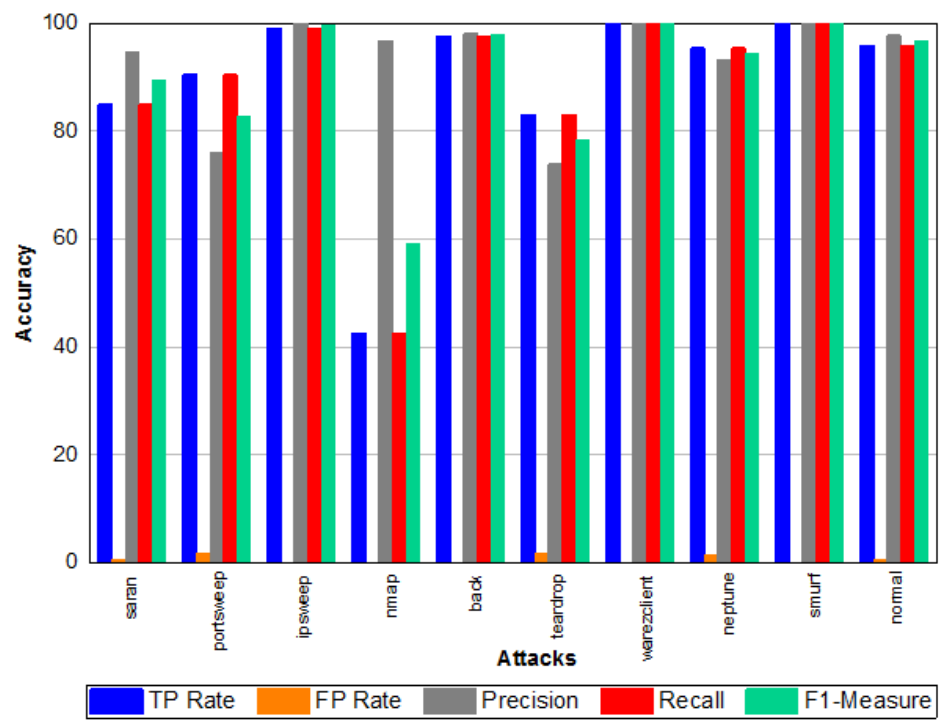

FIGURE 11 Classification report for K Nearest Neighbour

For the table 16, we can conclude that Saran attack, TP, FP, precision, recall and f1-score is $84.9 \%, 0.5 \%, 94.6 \%$, 84.9\%,89.5\% respectively. Portsweep has $90.50 \%, 1.9 \%, 76 \%, 90.50 \%$, and $82.60 \%$ TP, FP, precision, recall, f1-score respectively. TP rate for Ipswep, Back and Neptune attacks is $99.10 \%, 97.60 \%, 95.40 \%$, respectively. Similarly, FP rate for those attacks is $0 \%, 0.22,1.6 \%$, respectively. The precision for Ipsweep is $100 \%$. Recall and F1-Measure for Ipsweep is $99.10 \%, 99.50 \%$, respectively. Precision for Back and Neptune is $98.10 \% .93 .30 \%$, respectively. For back attack recall and f1-score is $97.60 \%, 97.90 \%$, respectively. Similarly, for Neptune, it is $95.40 \%$ and $94.30 \%$, 
respectively, for the Nmap TP rate and the recall score is $42.60 \%$, respectively. FR rate is $0 \%$. Precision and recall scores are $96.70 \%$ and $59.20 \%$, respectively. Warezclient and Smurf attack achieved promising results using the KNN classifier. Precision, recall, f1-score and TP rate are $100 \%$ respectively for both attacks. The normal class achieved, on average, 95\% TP, precision, recall and f1-score, respectively, depicted in figure 11.

TAB LE 17 Classification report for Neural Network

\begin{tabular}{llllll}
\hline Class & TP Rate \% & FP Rate \% & Precision \% & Recall \% & F1-score \% \\
\hline saran & 84.3 & 0.5 & 94.8 & 84.3 & 89.2 \\
portsweep & 91.8 & 5.9 & 51.1 & 91.8 & 65.7 \\
ipsweep & 99.1 & 0 & 99.4 & 99.1 & 99.2 \\
nmap & 42.6 & 0.1 & 90.6 & 42.6 & 58 \\
back & 98.7 & 1.1 & 88.6 & 98.7 & 93.4 \\
teardrop & 62.5 & 1 & 78.3 & 62.5 & 69.5 \\
warezclient & 100 & 0.1 & 99 & 100 & 99.5 \\
neptune & 96.7 & 1.6 & 93.3 & 96.7 & 95 \\
smurf & 100 & 0.1 & 99.7 & 100 & 99.8 \\
normal & 79.2 & 0.3 & 98.4 & 79.2 & 87.8 \\
& & & & & \\
\hline
\end{tabular}

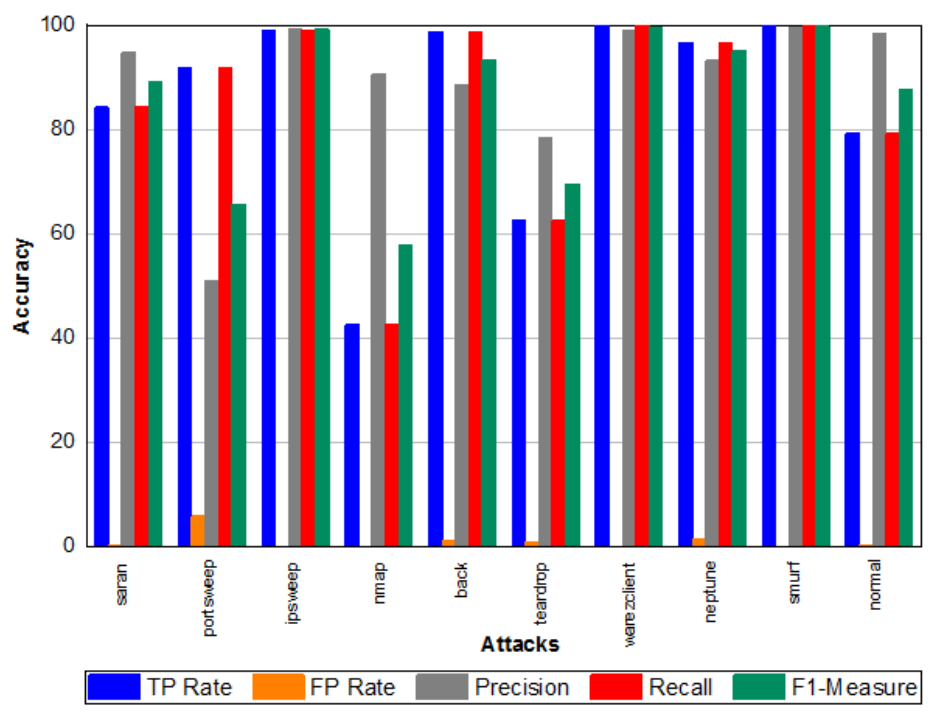

FIGURE 12 Classification report for Neural Network

From table 17 and figure 12, we conclude that the TR rate for attacks and the normal class is $95.36 \%$ on average. 
Similarly, the average FP rate is $1.06 \%$ for all the classes in NSLKDD dataset. Average precision, recall and F1-Measure scores are $89.32 \%, 85.49 \%, 85.33 \%$ respectively for all the attacks and normal class using decision tree algorithm.

\subsection{NSLKDD Multi Class Classification Experimental Results}

TAB LE 18 Classification report for Decision Report

\begin{tabular}{llllll}
\hline Class & TP Rate $\%$ & FP Rate \% & Precision \% & Recall \% & F1-score \% \\
\hline warezclient & 98.2 & 0.2 & 96 & 98.2 & 97.1 \\
ipsweep & 90.8 & 0.3 & 98.4 & 90.8 & 94.4 \\
portsweep & 99.2 & 0.1 & 99.1 & 99.2 & 99.1 \\
teardrop & 100 & 0 & 100 & 100 & 100 \\
nmap & 97.6 & 1.3 & 82.9 & 97.6 & 89.7 \\
smurf & 100 & 0.3 & 97.4 & 100 & 98.7 \\
back & 98.9 & 0 & 99.6 & 98.9 & 99.2 \\
satan & 98.8 & 0.2 & 98.7 & 98.8 & 98.8 \\
neptune & 99.3 & 0 & 99.8 & 99.3 & 99.6 \\
normal & 96.9 & 0.3 & 98.2 & 96.9 & 97.5 \\
\hline
\end{tabular}

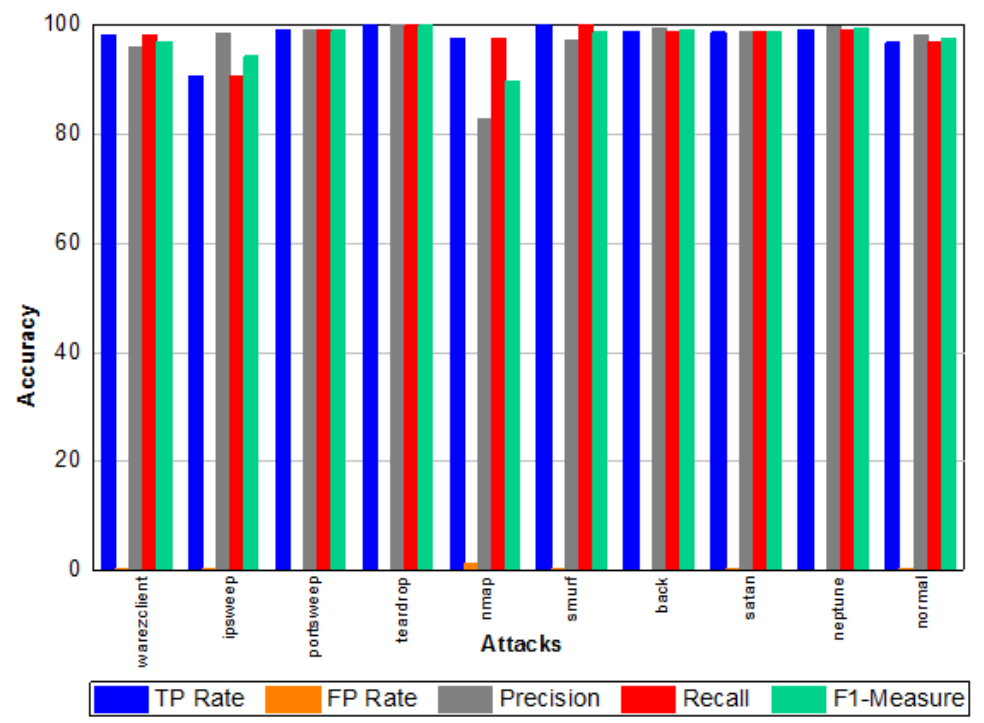

FIGURE 13 Classification report for Decision Tree 
From table 18 and figure 13, we can conclude that the TP rate for attacks and the normal class is $95.36 \%$ on average. Similarly, the average FP rate is $1.06 \%$ for all the classes in NSLKDD dataset. Average precision, recall and f1-measure scores are $89.32 \%, 85.49 \%, 85.33 \%$ respectively for all the attacks and normal class using decision tree algorithm.

TAB LE 19 Classification report for Random Forest

\begin{tabular}{llllll}
\hline Class & TP Rate $\%$ & FP Rate \% & Precision \% & Recall \% & F1-score \% \\
\hline warezclient & 100 & 0.1 & 98.6 & 100 & 99.3 \\
ipsweep & 90.8 & 0.1 & 99.3 & 90.8 & 94.9 \\
portsweep & 98.7 & 0.1 & 98.9 & 98.7 & 98.8 \\
teardrop & 100 & 0 & 100 & 100 & 100 \\
nmap & 96.7 & 1.3 & 83 & 96.7 & 89.4 \\
smurf & 100 & 0.3 & 97.9 & 100 & 99 \\
back & 100 & 0 & 100 & 100 & 100 \\
satan & 98.8 & 0.3 & 98.3 & 98.8 & 98.5 \\
neptune & 99.9 & 0.1 & 99.2 & 99.9 & 99.6 \\
normal & 98.4 & 0.2 & 99.1 & 98.4 & 98.7 \\
\hline
\end{tabular}

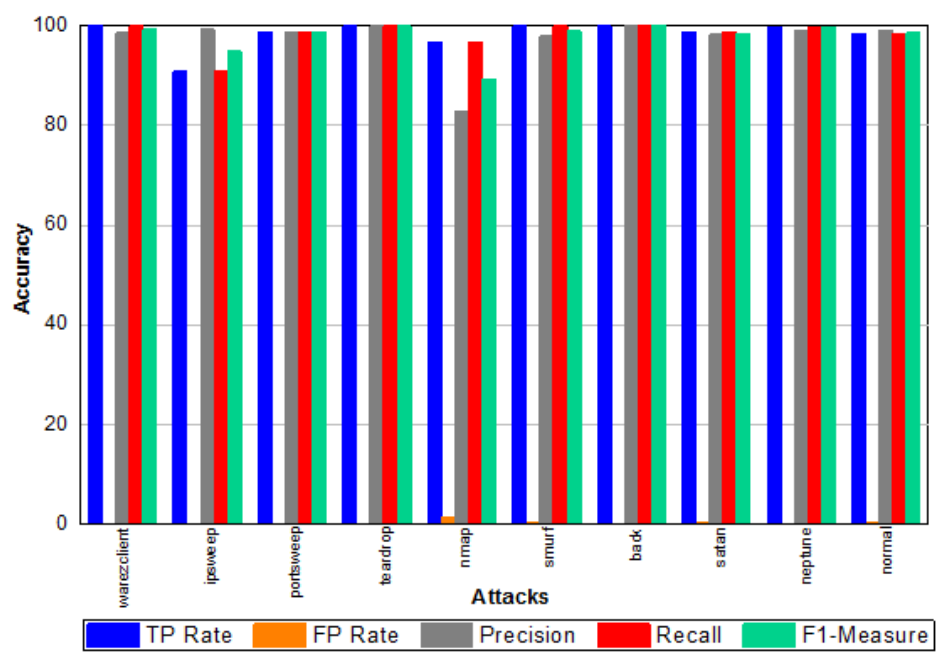

FIGURE 14 Classification report for Random Forest

Table 19 depicts that Warezclient, Teardrop, Smurf and Back attack have a 100\% TP rate and 100\% recall, respectively. Teardrop and Back attack has a 0\% FP rate, respectively. Warezlient, Ipsweep, Portsweep and Neptune have a $0.1 \%$ FP rate, respectively. Smurf and Satan have a $0.3 \%$ FP rate, respectively. Satan has $0.3 \%$ and normal has $0.2 \%$ FR rates, respectively. Warezclient, Portsweep and Satan have $98 \%$ precision, respectively. Ipsweerp, Neptune and 
normal have $99 \%$ precision, respectively. Portsweep, Neptune and normal class hs $98 \%$ recall, respectively. Similarly, Ipsweep, Nmap and Neptune have $90.8 \%, 96.7 \%$ and $99.9 \%$ recall, respectively. f1-measure for Warezclient, Smurf and Neptune is $99 \%$, respectively. Portsweep, Satan and Normal have $98 \%$ f1-measure, respectively. Teardrop and Back have $100 \% \mathrm{f1}$-measure, respectively. Nmap has $89.4 \% \mathrm{f1}$-measure using a random forest classifier and NSLKDD dataset. The visualization of these attacks is depicted in figure 14.

TA B LE 20 Classification report for K Nearest Neighbour

\begin{tabular}{llllll}
\hline Class & TP Rate \% & FP Rate \% & Precision \% & Recall \% & F1-score \% \\
\hline warezclient & 99.5 & 0.2 & 95.2 & 99.5 & 97.3 \\
ipsweep & 90.8 & 0.1 & 99.3 & 90.8 & 94.9 \\
portsweep & 97.9 & 0.1 & 99.1 & 97.9 & 98.5 \\
teardrop & 100 & 0 & 100 & 100 & 100 \\
nmap & 97 & 1.2 & 83.3 & 97 & 89.6 \\
smurf & 100 & 0.3 & 97.7 & 100 & 98.8 \\
back & 100 & 0 & 99.2 & 100 & 99.6 \\
satan & 98.7 & 0.4 & 97.7 & 98.7 & 98.2 \\
neptune & 99.3 & 0.2 & 98.8 & 99.3 & 99.1 \\
normal & 96.7 & 0.3 & 98.2 & 96.7 & 97.4 \\
\hline
\end{tabular}

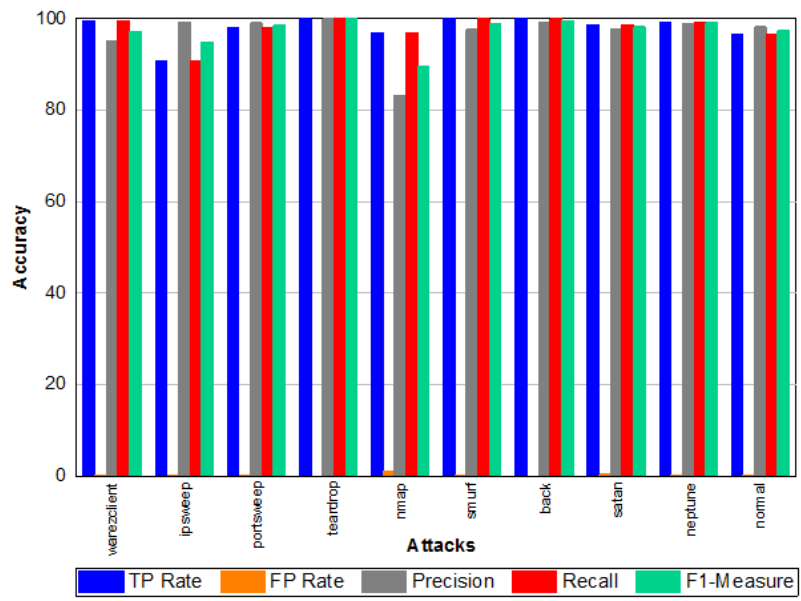

FIGURE 15 Classification report for K Nearest Neighbour

From table 20 and figure 15, we conclude that the TP rate for attacks and the normal class is $97.99 \%$ on average. Similarly, the average FP rate is $0.28 \%$ for all the classes in NSLKDD dataset. Average precision, recall and f1-measure scores are $97.97 \%, 97.99 \%$ and 97.34 respectively for all the attacks and normal class using KNN algorithm. 
TAB LE 21 Classification report for Neural Network

\begin{tabular}{llllll}
\hline Class & TP Rate $\%$ & FP Rate \% & Precision \% & Recall \% & F1-score \% \\
\hline warezclient & 99.5 & 0.5 & 88.6 & 99.5 & 93.7 \\
ipsweep & 90.5 & 0.2 & 99.1 & 90.5 & 94.6 \\
portsweep & 98.8 & 0.2 & 98.8 & 98.8 & 98.8 \\
teardrop & 100 & 0 & 100 & 100 & 100 \\
nmap & 91.1 & 1.3 & 82.2 & 91.1 & 86.4 \\
smurf & 100 & 0.3 & 97.4 & 100 & 98.7 \\
back & 98.5 & 7.2 & 37.7 & 98.5 & 54.6 \\
satan & 98.4 & 0.7 & 95.9 & 98.4 & 97.1 \\
neptune & 99.4 & 0.1 & 99.6 & 99.4 & 99.5 \\
normal & 46.7 & 0.4 & 94.9 & 46.7 & 62.6 \\
\hline
\end{tabular}

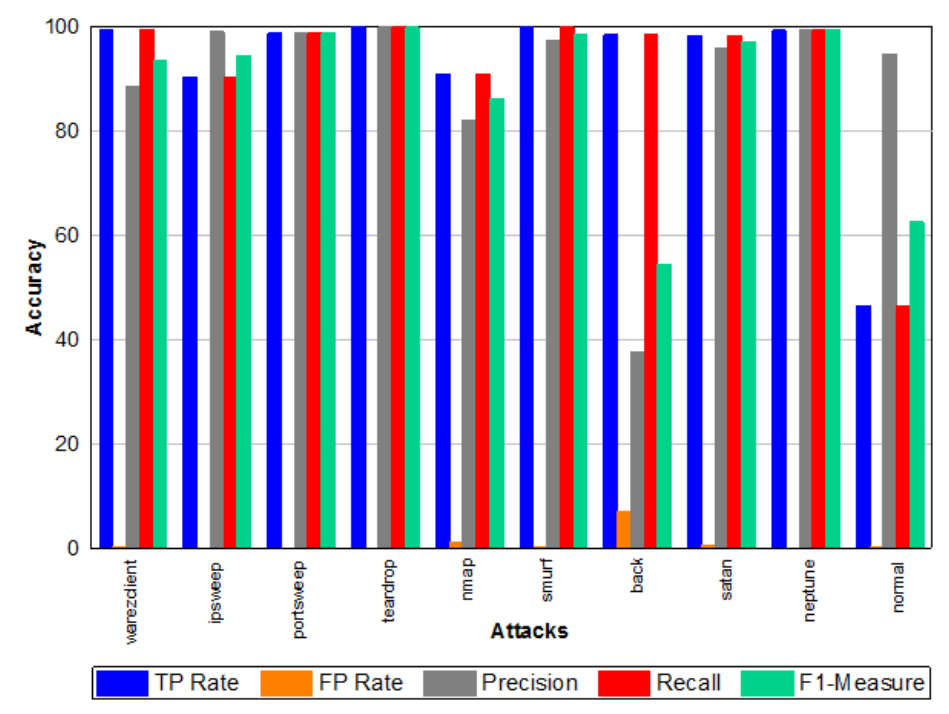

FIGURE 16 Classification report for Neural Network

From table 21 and figure 16, we conclude that the TP rate for attacks and the normal class is $92.29 \%$ on average. Similarly, the average FP rate is $1.6 \%$ for all the classes in NSLKDD dataset. Average precision, recall and F1-Measure scores becomes $89.44 \%, 92.25 \%$ and $88.6 \%$ respectively for all the attacks and normal class using decision tree algorithm. 
TAB LE 22 Comparison of proposed model with other models (KDD99)

\begin{tabular}{llll}
\hline Model & Accuracy \% & FAR \% & DR \% \\
\hline PSO+MCLP [32] & 99.13 & 1.94 & - \\
TVCPSO [41] & - & 0.80 & 97 \\
SVM-ELM [63] & 95.75 & 1.87 & 95.17 \\
PSO [64] & 88.5 & - & - \\
DNN [65] & 75.5 & 0.85 & 76 \\
PSO-ANN [66] & 92.5 & - & - \\
ANN(FNN-LSO) & 94.02 & 2.23 & 89.83 \\
Proposed Model (PSO+NN) & 99.20 & 0.5 & 99.70 \\
\hline
\end{tabular}

TAB LE 23 Comparison of proposed model with other models (NSLKDD)

\begin{tabular}{llll}
\hline Model & Accuracy $\%$ & FAR $\%$ & DR \% \\
\hline RF [43] & 93.77 & - & - \\
SVM-ELM [44] & 95.75 & 1.87 & 95.17 \\
DNEDRON [45] & 97.55 & 1.08 & 95.97 \\
RNN-IDS [46] & 99.81 & 5.09 & 96.92 \\
HIERARCHICAL SOM [47] & - & 2.19 & 93.46 \\
ADABOOST [48] & - & 3.14 & 91.20 \\
LSTM [49] & 93.82 & 0.09 & 77.12 \\
GA [50] & 88.77 & - & - \\
Proposed Model & 99.65 & 0.08 & 99.3 \\
\hline
\end{tabular}

\section{5 | CONCLUSION AND FUTURE WORK}

This paper proposes a feature selection base IDS system for smart grid systems. For this purpose, we have used weighted PSO to improve the false alarm rate in the IDS. Optimal features are selected from KDD99 and NSLKDD datasets. After optimal features selection, these features are passed to machine learning models. We have applied various machine learning algorithms on NSLKDD and KDD99 datasets during the experiments. After the collection of datasets, we have transformed them into a binary classification: attack class and normal class as well as we used multiple attacks. 9 attacks are used for the KDD99 dataset. In comparison, 21 attacks are used for the NSLKDD dataset. Initially, we have performed preprocessing on the datasets and non-numeric values are replaced with numeric encoding. Next, the data is normalized using min-max normalization. After that, we have performed feature selection using particle swarm optimization and selected the best features. After feature selection, we have applied different machine learning algorithms on both the datasets. Random Forest and Neural Network have outperformed all other 
methods in terms of accuracy, training time and false alarm rate. We have also compared our proposed methodology with other recent work as shown in Table 22 and Table 23. Experimental results prove that our method performs better in terms of detection rate, false alarm rate and accuracy for both KDD99 and NSLKDD datasets. In future, we intend to repeat this experiment with multiple classes with feature selection methods using deep learning algorithms.

\section{references}

[1] Otoum Y, Liu D, Nayak A. DL-IDS: a deep learning-based intrusion detection framework for securing loT. Transactions on Emerging Telecommunications Technologies 2019;p. e3803.

[2] Yousaf A, Loan A, Babiceanu RF, Yousaf O. Physical-layer intrusion detection system for smart jamming attacks. Transactions on Emerging Telecommunications Technologies 2017;28(11):e3189.

[3] Irshad O, Khan MUG, Iqbal R, Basheer S, Bashir AK. Performance optimization of loT based biological systems using deep learning. Computer Communications 2020;.

[4] Vora J, Kaneriya S, Tanwar S, Tyagi S, Kumar N, Obaidat M. TILAA: Tactile Internet-based ambient assistant living in fog environment. Future Generation Computer Systems 2019;98:635-649.

[5] Uppal HAM, Javed M, Arshad M. An overview of intrusion detection system (IDS) along with its commonly used techniques and classifications. International Journal of Computer Science and Telecommunications 2014;5(2):20-24.

[6] Bhattacharya S, Kaluri R, Singh S, Alazab M, Tariq U, et al. A Novel PCA-Firefly based XGBoost classification model for Intrusion Detection in Networks using GPU. Electronics 2020;9(2):219.

[7] Alazab M, Khan S, Siva Rama Krishnan S, Pham Q, Praveen Kumar Reddy M, Gadekallu TR. A Multidirectional LSTM Model for Predicting the Stability of a Smart Grid. IEEE Access 2020;.

[8] Almseidin M, Alzubi M, Kovacs S, Alkasassbeh M. Evaluation of machine learning algorithms for intrusion detection system. In: 2017 IEEE 15th International Symposium on Intelligent Systems and Informatics (SISY) IEEE; 2017. p. 000277000282.

[9] Iwendi C, Maddikunta PKR, Gadekallu TR, Lakshmanna K, Bashir AK, Piran MJ. A metaheuristic optimization approach for energy efficiency in the loT networks. Software: Practice and Experience 2020;.

[10] Jyothsna V, Prasad VR, Prasad KM. A review of anomaly based intrusion detection systems. International Journal of Computer Applications 2011;28(7):26-35.

[11] Kaur H, Kumar N, Batra S. ClaMPP: a cloud-based multi-party privacy preserving classification scheme for distributed applications. The Journal of Supercomputing 2019;75(6):3046-3075.

[12] Aujla GS, Kumar N, Singh M, Zomaya AY. Energy trading with dynamic pricing for electric vehicles in a smart city environment. Journal of Parallel and Distributed Computing 2019;127:169-183.

[13] Aghdam MH, Ghasem-Aghaee N, Basiri ME. Application of ant colony optimization for feature selection in text categorization. In: 2008 IEEE Congress on Evolutionary Computation (IEEE World Congress on Computational Intelligence) IEEE; 2008. p. 2867-2873.

[14] Aghdam MH, Tanha J, Naghsh-Nilchi AR, Basiri ME. Combination of ant colony optimization and Bayesian classification for feature selection in a bioinformatics dataset. Journal of Computer Science \& Systems Biology 2009;2(3):186-199.

[15] Nguyen VG, Brunstrom A, Grinnemo KJ, Taheri J, Liyanage M, Ahmad I, et al. 5 G mobile networks: Requirements, enabling technologies, and research activities. A Comprehensive Guide to 5G Security 2018;p. 31-57. 
[16] Qasim OS, Algamal ZY. Feature selection using particle swarm optimization-based logistic regression model. Chemometrics and Intelligent Laboratory Systems 2018;182:41-46.

[17] Ma T, Xu C, Zhou Z, Kuang X, Zhong L. SE-PSO: Resource Scheduling Strategy for Multimedia Cloud Platform Based on Security Enhanced Virtual Migration. In: 2019 15th International Wireless Communications \& Mobile Computing Conference (IWCMC) IEEE; 2019. p. 650-655.

[18] Iwendi C, Khan S, Anajemba JH, Mittal M, Alenezi M, Alazab M. The Use of Ensemble Models for Multiple Class and Binary Class Classification for Improving Intrusion Detection Systems. Sensors 2020;20(9):2559.

[19] Xue B, Zhang M, Browne WN. Particle swarm optimization for feature selection in classification: A multi-objective approach. IEEE transactions on cybernetics 2012;43(6):1656-1671.

[20] Xue B, Zhang M, Browne WN. Particle swarm optimisation for feature selection in classification: Novel initialisation and updating mechanisms. Applied soft computing 2014;18:261-276.

[21] Reddy T, RM SP, Parimala M, Chowdhary CL, Hakak S, Khan WZ, et al. A deep neural networks based model for uninterrupted marine environment monitoring. Computer Communications 2020;.

[22] Revathi S, Malathi A. A detailed analysis on NSL-KDD dataset using various machine learning techniques for intrusion detection. International Journal of Engineering Research \& Technology (IJERT) 2013;2(12):1848-1853.

[23] Tavallaee M, Bagheri E, Lu W, Ghorbani AA. A detailed analysis of the KDD CUP 99 data set. In: 2009 IEEE symposium on computational intelligence for security and defense applications IEEE; 2009. p. 1-6.

[24] Liu G, Yi Z, Yang S. A hierarchical intrusion detection model based on the PCA neural networks. Neurocomputing 2007;70(7-9):1561-1568.

[25] Heba FE, Darwish A, Hassanien AE, Abraham A. Principle components analysis and support vector machine based intrusion detection system. In: 2010 10th international conference on intelligent systems design and applications IEEE; 2010. p. 363-367.

[26] Chae Hs, Jo Bo, Choi SH, Park Tk. Feature selection for intrusion detection using NSL-KDD. Recent advances in computer science 2013;p. 184-187.

[27] Ahmad I, Hussain M, Alghamdi A, Alelaiwi A. Enhancing SVM performance in intrusion detection using optimal feature subset selection based on genetic principal components. Neural computing and applications 2014;24(7-8):1671-1682.

[28] Manekar V, Waghmare K. Intrusion detection system using support vector machine (SVM) and particle swarm optimization (PSO). International Journal of Advanced Computer Research 2014;4(3):808.

[29] Tong L, Wu Q. Intrusion Feature Selection Algorithm Based on Particle Swarm Optimization. International Journal of Computer Science and Network Security (IJCSNS) 2014;14(12):40.

[30] Zhang T, Kuang X, Zhou Z, Gao H, Xu C. An Intelligent Route Mutation Mechanism against Mixed Attack Based on Security Awareness. In: 2019 IEEE Global Communications Conference (GLOBECOM) IEEE; 2019. p. 1-6.

[31] Patel R, Bakhshi D, Arjariya T. Random particle swarm optimization (RPSO) based intrusion detection system. International Journal of Advanced Technology and Engineering Exploration 2015;2(5):60.

[32] Bamakan SMH, Amiri B, Mirzabagheri M, Shi Y. A new intrusion detection approach using PSO based multiple criteria linear programming. Procedia Computer Science 2015;55:231-237.

[33] Syarif AR, Gata W. Intrusion detection system using hybrid binary PSO and K-nearest neighborhood algorithm. In: 2017 11th International Conference on Information \& Communication Technology and System (ICTS) IEEE; 2017. p. $181-186$. 
[34] Mukherjee S, Sharma N. Intrusion detection using naive Bayes classifier with feature reduction. Procedia Technology 2012;4:119-128.

[35] Tesfahun A, Bhaskari DL. Intrusion detection using random forests classifier with SMOTE and feature reduction. In: 2013 International Conference on Cloud \& Ubiquitous Computing \& Emerging Technologies IEEE; 2013. p. $127-132$.

[36] Shrivas AK, Dewangan AK. An ensemble model for classification of attacks with feature selection based on KDD99 and NSL-KDD data set. International Journal of Computer Applications 2014;99(15):8-13.

[37] Ahmad I. Feature selection using particle swarm optimization in intrusion detection. International Journal of Distributed Sensor Networks 2015;11(10):806954.

[38] Eesa AS, Orman Z, Brifcani AMA. A novel feature-selection approach based on the cuttlefish optimization algorithm for intrusion detection systems. Expert Systems with Applications 2015;42(5):2670-2679.

[39] Rai K, Devi MS, Guleria A. Decision tree based algorithm for intrusion detection. International Journal of Advanced Networking and Applications 2016;7(4):2828.

[40] Ambusaidi MA, He X, Nanda P, Tan Z. Building an intrusion detection system using a filter-based feature selection algorithm. IEEE transactions on computers 2016;65(10):2986-2998.

[41] Bamakan SMH, Wang H, Yingjie T, Shi Y. An effective intrusion detection framework based on MCLP/SVM optimized by time-varying chaos particle swarm optimization. Neurocomputing 2016;199:90-102.

[42] Thaseen IS, Kumar CA. Intrusion detection model using fusion of chi-square feature selection and multi class SVM. Journal of King Saud University-Computer and Information Sciences 2017;29(4):462-472.

[43] Pajouh HH, Javidan R, Khayami R, Ali D, Choo KKR. A two-layer dimension reduction and two-tier classification model for anomaly-based intrusion detection in loT backbone networks. IEEE Transactions on Emerging Topics in Computing 2016;.

[44] Shone N, Ngoc TN, Phai VD, Shi Q. A deep learning approach to network intrusion detection. IEEE Transactions on Emerging Topics in Computational Intelligence 2018;2(1):41-50.

[45] Naseer S, Saleem Y, Khalid S, Bashir MK, Han J, lqbal MM, et al. Enhanced network anomaly detection based on deep neural networks. IEEE Access 2018;6:48231-48246.

[46] Sakr MM, Tawfeeq MA, El-Sisi AB. Network Intrusion Detection System based PSO-SVM for Cloud Computing. International Journal of Computer Network and Information Security 2019;11(3):22.

[47] Woo Jh, Song JY, Choi YJ. Performance Enhancement of Deep Neural Network Using Feature Selection and Preprocessing for Intrusion Detection. In: 2019 International Conference on Artificial Intelligence in Information and Communication (ICAIIC) IEEE; 2019. p. 415-417.

[48] Cadima J, Cerdeira JO, Minhoto M. Computational aspects of algorithms for variable selection in the context of principal components. Computational statistics \& data analysis 2004;47(2):225-236.

[49] Greselin F, Zitikis R. From the classical Gini index of income inequality to a new Zenga-type relative measure of risk: A modeller's perspective. Econometrics 2018;6(1):4.

[50] Goldberg DE, Holland JH. Genetic algorithms and machine learning 1988;.

[51] Bai Q. Analysis of particle swarm optimization algorithm. Computer and information science 2010;3(1):180.

[52] Kayacik HG, Zincir-Heywood AN, Heywood MI. Selecting features for intrusion detection: A feature relevance analysis on KDD 99 intrusion detection datasets. In: Proceedings of the third annual conference on privacy, security and trust, vol. 94; 2005. p. 1723-1722. 
[53] Nykvist C, Larsson M, Sodhro AH, Gurtov A. A lightweight portable intrusion detection communication system for auditing applications. International Journal of Communication Systems 2020;p. e4327.

[54] Kennedy J, Eberhart R. Particle swarm optimization. In: Proceedings of ICNN'95-International Conference on Neural Networks, vol. 4 IEEE; 1995. p. 1942-1948.

[55] Shi Y, Eberhart R. A modified particle swarm optimizer. In: 1998 IEEE international conference on evolutionary computation proceedings. IEEE world congress on computational intelligence (Cat. No. 98TH8360) IEEE; 1998. p. 69-73.

[56] Ahmad I, e Amin F. Towards feature subset selection in intrusion detection. In: 2014 IEEE 7th Joint International Information Technology and Artificial Intelligence Conference IEEE; 2014. p. 68-73.

[57] Bre F, Gimenez JM, Fachinotti VD. Prediction of wind pressure coefficients on building surfaces using artificial neural networks. Energy and Buildings 2018;158:1429-1441.

[58] Quinlan J. Program for machine learning. C4 5 1993;.

[59] Kim G, Lee S, Kim S. A novel hybrid intrusion detection method integrating anomaly detection with misuse detection. Expert Systems with Applications 2014;41(4):1690-1700.

[60] Mulay SA, Devale P, Garje G. Intrusion detection system using support vector machine and decision tree. International Journal of Computer Applications 2010;3(3):40-43.

[61] Shakil M, Fuad Yousif Mohammed A, Arul R, Bashir AK, Choi JK. A novel dynamic framework to detect DDoS in SDN using metaheuristic clustering. Transactions on Emerging Telecommunications Technologies 2019;p. e3622.

[62] Iwendi C, Khan S, Anajemba JH, Bashir AK, Noor F. Realizing an efficient loMT-assisted patient diet recommendation system through machine learning model. IEEE Access 2020;8:28462-28474.

[63] Peng K, Leung V, Zheng L, Wang S, Huang C, Lin T. Intrusion detection system based on decision tree over big data in fog environment. Wireless Communications and Mobile Computing 2018;2018.

[64] Chung YY, Wahid N. A hybrid network intrusion detection system using simplified swarm optimization (SSO). Applied soft computing 2012;12(9):3014-3022.

[65] Tang TA, Mhamdi L, McLernon D, Zaidi SAR, Ghogho M. Deep learning approach for network intrusion detection in software defined networking. In: 2016 International Conference on Wireless Networks and Mobile Communications (WINCOM) IEEE; 2016. p. 258-263.

[66] Dash T. A study on intrusion detection using neural networks trained with evolutionary algorithms. Soft Computing 2017;21(10):2687-2700.

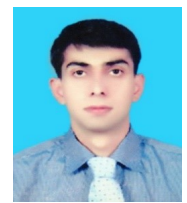

Suleman Khan Suleman Khan received the master's degree from the Department of Computer Science, Air University Islamabad, in 2019. He is currently a Research Associate with Air University, Pakistan. His research interests include network security, machine learning, and data science. 


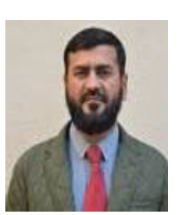

Dr. Kashif Kifayat received his Ph.D. in Cyber Security from Liverpool John Moores University, Liverpool, UK, in 2008. He is currently working as Professor and Chair of Cyber Security Department at Air University, Islamabad, Pakistan. Prior to this, he was Reader in Cyber Security at Liverpool John Moores University, UK. His current research interests include network security, security of complex systems, intrusion detection, secure service composition, privacy-preserving data aggregation, cryptography, computer forensics and loT security. He has published around 90 papers in international conference proceedings and journals and served in a number of conferences IPCs and journal editorial boards. He has also played a key role in many funded research and development projects related to his research topics.

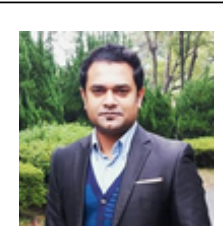

Dr.Ali Kashif Bashir (Senior Member, IEEE) received the B.S. degree from the University of Management and Technology, Pakistan, the M.S. degree from Ajou University, South Korea, and the Ph.D. degree in computer science and engineering from Korea University, South Korea. He is currently a Senior Lecturer with the School of Computing, Mathematics, and Digital Technology, Manchester Metropolitan University, U.K. He is also a Distinguished Speaker of ACM. His past assignments include an Associate Professor of information and communication technologies with the Faculty of Science and Technology, University of the Faroe Islands, Denmark; Osaka University, Japan; the Nara National College of Technology, Japan; the National Fusion Research Institute, South Korea; Southern Power Company Ltd., South Korea; and the Seoul Metropolitan Government, South Korea. He is the author of over 80 peer-reviewed articles. He is supervising/co-supervising several graduate (M.S. and Ph.D.) students. His research interests include the Internet of Things, wireless networks, distributed systems, network/cyber security, and cloud/network function virtualization. Dr. Bashir has served as the Program Chair, the Publicity Chair, and the Track Chair on several conferences and workshops. He has delivered several invited and keynote talks, and reviewed the technology leading articles for journals like the IEEE Transactions on Industrial Informatics, the IEEE Communication Magazine, the IEEE Communication Letters, the IEEE Internet of Things, and the IEICE Journals, and conferences, such as the IEEE Infocom, the IEEE ICC, the IEEE Globecom, and the IEEE Cloud of Things. He is also serving as the Editor-in-Chief for the IEEE Future Directions Newsletter. He is also an Editor of several journals and also has served/serving as a Guest Editor on several special issues in journals of IEEE, Elsevier, and Springer.

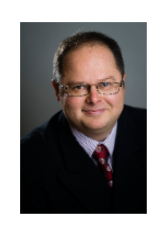

Dr.Andrei Gurtov,(Senior Member, IEEE) is a Professor of Computer Science at Linköping University, Sweden. Previously he was at University of Oulu (3 years) and Aalto University (6 years) and visiting the International Computer Science Institute at Berkeley multiple times. He received his M.Sc (2000) and Ph.D. (2004) degrees in Computer Science from the University of Helsinki, Finland. Prof. Gurtov co-authored over 200 publications, including 4 books, 5 IETF RFCs, 6 patents, over 60 journal and 110 conference articles. He supervised $15 \mathrm{PhD}$ theses. Professor Gurtov's research interests are in network 
protocols, security of vehicular, airborne, industrial systems, mobile, wireless and loT networks, SmartGrids. He is an ACM Distinguished Scientist, IEEE ComSoc Distinguished Lecturer and Vice-chair of IEEE Sweden section. He received best paper awards at IEEE CSCN'17 and IEEE Globecom'11, was co-adviser of the best Doctoral Thesis in CS in Finland in 2017. He had served on numerous journal editorial boards and conference program committees, including IEEE Internet of Things journal, MDPI Sensors, IEEE ICNP, ACM MSWiM, and IFIP Networking. URL: http://gurtov.com.

Dr.Mehdi hassan has done his PhD in the area of intelligent disease diagnosis using medical imaging.
He earned his PhD degree from PIEAS Pakistan in 2015. He has published several top rank interna-
tional journal and conference papers. He has been working in Artificial Intelligence specifically deep
neural networks, machine learning and image processing. He has supervised several MS students
$\mathrm{He}$ is Co-PI in national center of excellence in cyber security lab. Currently, he is serving as Chair
Department of Computer Science at Air University.

